\title{
Interpolating Sequences of Parabolic Bergman Spaces
}

\author{
Masaharu Nishio • Noriaki Suzuki • Masahiro Yamada
}

Received: 3 October 2007 / Accepted: 12 March 2008 / Published online: 17 April 2008

(C) The Author(s) 2008

\begin{abstract}
The parabolic Bergman space is a Banach space of $L^{p}$-solutions of some parabolic equations on the upper half-space $H$. We study interpolating theorem for these spaces. It is shown that if a sequence in $H$ is $\delta$-separated with $\delta$ sufficiently near 1 , then it interpolates on parabolic Bergman spaces.
\end{abstract}

Keywords Bergman space - Interpolating sequence $\cdot$ Parabolic operator of fractional order

Mathematics Subject Classifications (2000) Primary 35K05 • Secondary 26D10 • 31B10

\section{Introduction}

Let $H$ be the upper half-space of the $(n+1)$-dimensional Euclidean space $\mathbb{R}^{n+1}(n \geq 1)$, that is, $H=\left\{X=(x, t) \in \mathbb{R}^{n+1} ; x=\left(x_{1}, \ldots, x_{n}\right) \in \mathbb{R}^{n}, t>0\right\}$. For $1 \leq p<\infty$, the

This work was supported in part by Grant-in-Aid for Scientific Research (C) No.18540168, No.18540169, and No.19540193, Japan Society for the Promotion of Science.

\footnotetext{
M. Nishio

Department of Mathematics, Osaka City University, Sugimoto, Sumiyoshi 3-3-138, 558-8585 Osaka, Japan

e-mail:nishio@sci.osaka-cu.ac.jp

N. Suzuki

Graduate School of Mathematics, Nagoya University,

Chikusa-ku, 464-8602 Nagoya, Japan

e-mail: nsuzuki@math.nagoya-u.ac.jp

M. Yamada $(\varangle)$

Department of Mathematics, Faculty of Education, Gifu University,

Yanagido 1-1, 501-1193 Gifu, Japan

e-mail: yamada33@gifu-u.ac.jp
} 
Lebesgue space $L^{p}=L^{p}(H, d V)$ is defined to be a Banach space of Lebesgue measurable functions on $H$ with

$$
\|u\|_{p}:=\left(\int_{H}|u(X)|^{p} d V(X)\right)^{1 / p}=\left(\int_{H}|u(x, t)|^{p} d V(x, t)\right)^{1 / p}<\infty,
$$

where $d V$ is the Lebesgue measure on $H$. For $0<\alpha \leq 1$, let $L^{(\alpha)}$ denote a parabolic operator

$$
L^{(\alpha)}:=\frac{\partial}{\partial t}+(-\Delta)^{\alpha}
$$

where $\Delta=\Delta_{x}=\frac{\partial^{2}}{\partial x_{1}^{2}}+\cdots+\frac{\partial^{2}}{\partial x_{n}^{2}}$. A continuous function $u$ on $H$ is said to be $L^{(\alpha)}$ harmonic if $L^{(\alpha)} u=0$ in the sense of distributions. (For details, see Section 2 of [7].) The parabolic Bergman space $\boldsymbol{b}_{\alpha}^{p}$ is the set of all $L^{(\alpha)}$-harmonic functions on $H$ which belong to $L^{p}$, and it is a Banach space with the $L^{p}$ norm. It is known that $\boldsymbol{b}_{\alpha}^{p} \subset$ $C^{\infty}(H)$ (see Theorem 5.4 of [7]), and when $\alpha=1 / 2, \boldsymbol{b}_{1 / 2}^{p}$ coincide with harmonic Bergman spaces studied by Ramey and Yi [11] (see Corollary 4.4 of [7] and Section 3 of [8]).

In this paper, we study interpolating sequences of parabolic Bergman spaces. Let $\left\{X_{j}\right\}=\left\{\left(x_{j}, t_{j}\right)\right\}$ be a sequence in $H$. For $u \in \boldsymbol{b}_{\alpha}^{p}$, we define a sequence of real numbers $T_{p} u$ by

$$
T_{p} u:=\left\{t_{j}^{\left(\frac{n}{2 \alpha}+1\right) \frac{1}{p}} u\left(X_{j}\right)\right\} .
$$

We say that $\left\{X_{j}\right\}$ is a $\boldsymbol{b}_{\alpha}^{p}$-interpolating sequence if $T_{p} u \in \ell^{p}$ for all $u \in \boldsymbol{b}_{\alpha}^{p}$ and the linear operator $T_{p}: \boldsymbol{b}_{\alpha}^{p} \rightarrow \ell^{p}$ is bounded and onto. Here $\ell^{p}$ is the Banach space of all $p$-th summable sequences. It is known that there exists a constant $C>0$ such that

$$
t^{\left(\frac{n}{2 \alpha}+1\right) \frac{1}{p}}|u(x, t)| \leq C\|u\|_{p}
$$

for all $u \in \boldsymbol{b}_{\alpha}^{p}$ and all $(x, t) \in H$ (Proposition 5.2 of [7]). This is a reason why we consider a weight $t_{j}^{\left(\frac{n}{2 \alpha}+1\right) \frac{1}{p}}$ in the sequence in Eq. 1. Our result of this paper can be applied to study an analysis of Carleson measures and Toeplitz operators on parabolic Bergman spaces, whose applications will be described elsewhere.

Interpolations of holomorphic Bergman spaces were studied on various settings and well known. It is a result of Amar [1] (see also [12]) that if a sequence of points in the unit ball of $\mathbb{C}^{n}$ is separated enough with respect to the pseudo-hyperbolic distance then the sequence is an interpolating sequence of holomorphic Bergman spaces (the results of [1] and [12] are more general). Seip [13] gave a characterization of interpolating sequences of Bergman spaces on the unit disk. For the case of several variables, Marco and Massaneda [6] showed that Seip's condition for a sequence in the unit disk is sufficient to be of interpolating, however a complete generalization of Seip's characterization is not known yet. As for harmonic case, Choe and Yi [3] studied interpolations of harmonic Bergman spaces on the upper half-space of $\mathbb{R}^{n+1}$ and obtained a result similar to Amar. The result of Amar (or Choe and Yi) is useful and available for studying Carleson measures on Bergman spaces, and applied examples were known (see [5] and Theorem 4.4 of [4]). Our study is inspired by [3], but the details are different, because the parabolic operator $L^{(\alpha)}$ is not a local operator. 
Since the fundamental solution $W^{(\alpha)}$ of $L^{(\alpha)}$ plays an important role in our arguments, we present some properties of $W^{(\alpha)}$ and its related kernels in Section 2. The interpolation theorem in [3] is described by the pseudo-hyperbolic distance on the upper half-space, however, it is difficult to define a suitable distance in our case. For this we use parabolic cylinders and $\delta$-separated sequences in the parabolic sense and discuss them in Section 3. Our main theorem is stated in Section 4. It says that if a sequence is $\delta$-separated with $\delta$ sufficiently near 1 , then it is an interpolating sequence (of general order) for parabolic Bergman spaces. In Section 5, we give a corresponding result for parabolic Bloch spaces and parabolic little Bloch spaces.

Throughout this paper, $C=C(a, b, \cdots)$ will denote a positive constant which is depending only on $a, b, \cdots$, and not necessarily the same at each occurrence.

\section{Preliminaries}

In this section, we describe some basic results concerning derivatives of the fundamental solution of $L^{(\alpha)}$. For $x \in \mathbb{R}^{n}$, let

$$
W^{(\alpha)}(x, t)= \begin{cases}\frac{1}{(2 \pi)^{n}} \int_{\mathbb{R}^{n}} \exp \left(-t|\xi|^{2 \alpha}+i x \cdot \xi\right) d \xi & t>0 \\ 0 & t \leq 0,\end{cases}
$$

where $x \cdot \xi$ denotes the inner product on $\mathbb{R}^{n}$ and $|\xi|=(\xi \cdot \xi)^{1 / 2}$. The function $W^{(\alpha)}$ is the fundamental solution of $L^{(\alpha)}$ and $L^{(\alpha)}$-harmonic on $H$. Let $\gamma=\left(\gamma_{1}, \cdots, \gamma_{n}\right) \in$ $\mathbb{N}_{0}^{n}$ be a multi-index and $k \in \mathbb{N}_{0}$, where $\mathbb{N}_{0}=\mathbb{N} \cup\{0\}$. We use the notation $\partial_{x}^{\gamma} \partial_{t}^{k}=$ $\partial_{x_{1}}^{\gamma_{1}} \cdots \partial_{x_{n}}^{\gamma_{n}} \partial_{t}^{k}=\partial^{|\gamma|+k} / \partial x_{1}^{\gamma_{1}} \cdots \partial x_{n}^{\gamma_{n}} \partial t^{k}$, where $|\gamma|:=\gamma_{1}+\cdots+\gamma_{n}$. Then, by Eq. 2 , the inductive method implies that

$$
\partial_{x}^{\gamma} \partial_{t}^{k} W^{(\alpha)}(x, t)=t^{-\frac{n+|\gamma|}{2 \alpha}-k}\left(\partial_{x}^{\gamma} \partial_{t}^{k} W^{(\alpha)}\right)\left(t^{-1 / 2 \alpha} x, 1\right) .
$$

The following estimate is Lemma 1 of [9]: There exists a constant $C=C(n, \alpha, \gamma, k)>$ 0 such that

$$
\left|\partial_{x}^{\gamma} \partial_{t}^{k} W^{(\alpha)}(x, t)\right| \leq \frac{C}{\left(t+|x|^{2 \alpha}\right)^{\frac{n+|\gamma|}{2 \alpha}+k}}
$$

for all $(x, t) \in H$. The $\alpha$-parabolic Bergman kernel $R_{\alpha}(X, Y)=R_{\alpha}(x, t ; y, s)$ is given by

$$
R_{\alpha}(x, t ; y, s):=-2 \partial_{t} W^{(\alpha)}(x-y, t+s)=-2 \partial_{s} W^{(\alpha)}(x-y, t+s) .
$$

This is the reproducing kernel of $\boldsymbol{b}_{\alpha}^{2}$. Moreover it has the reproducing property for all $\boldsymbol{b}_{\alpha}^{p}$ (see Theorem 6.3 of [7]), that is,

$$
u(x, t)=\int_{H} u(y, s) R_{\alpha}(x, t ; y, s) d V(y, s)
$$

for all $(x, t) \in H$ and $u \in \boldsymbol{b}_{\alpha}^{p}$ with $1 \leq p<\infty$. We also use the kernel $R_{\alpha}^{\gamma, k}(X, Y)=$ $R_{\alpha}^{\gamma, k}(x, t ; y, s)$ defined by

$$
R_{\alpha}^{\gamma, k}(x, t ; y, s):=c_{k} s^{\frac{|\gamma|}{2 \alpha}+k} \partial_{x}^{\gamma} \partial_{t}^{k} R_{\alpha}(x, t ; y, s)=(-1)^{|\gamma|} c_{k} s^{\frac{|\gamma|}{2 \alpha}+k} \partial_{y}^{\gamma} \partial_{s}^{k} R_{\alpha}(x, t ; y, s),
$$

where $c_{k}=(-2)^{k} / k !$. Note that $R_{\alpha}(X, Y)=R_{\alpha}(Y, X)$, but $R_{\alpha}^{\gamma, k}(X, Y) \neq R_{\alpha}^{\gamma, k}(Y, X)$ in general. 
Now we define an $\alpha$-parabolic cylinder, which will be used for the definition of separated sequences in Section 3. For $Y=(y, s) \in H$ and $0<\delta<1$, an $\alpha$-parabolic cylinder $S_{\delta}^{(\alpha)}(Y)=S_{\delta}^{(\alpha)}(y, s)$ is defined by

$$
\begin{aligned}
S_{\delta}^{(\alpha)}(y, s) & :=\left\{(x, t) \in H ;|x-y|<\left(\frac{2 \delta}{1-\delta^{2}} s\right)^{1 / 2 \alpha},\left|t-\frac{1+\delta^{2}}{1-\delta^{2}} s\right|<\frac{2 \delta}{1-\delta^{2}} s\right\} \\
& =\left\{(x, t) \in H ;|x-y|<\left(\frac{2 \delta}{1-\delta^{2}} s\right)^{1 / 2 \alpha}, \frac{1-\delta}{1+\delta} s<t<\frac{1+\delta}{1-\delta} s\right\} .
\end{aligned}
$$

Clearly $\lim _{\delta \rightarrow 1} S_{\delta}^{(\alpha)}(Y)=H$ and $S_{\delta}^{(\alpha)}(y, s)=\Phi_{Y}^{(\alpha)}\left(S_{\delta}^{(\alpha)}(0,1)\right)$, where $\Phi_{Y}^{(\alpha)}(X)$ is the function defined by

$$
\Phi_{Y}^{(\alpha)}(X):=\left(s^{1 / 2 \alpha} x+y, s t\right)
$$

for $X=(x, t) \in H$. Also $V\left(S_{\delta}^{(\alpha)}(y, s)\right)=2 B_{n}\left(\frac{2 \delta}{1-\delta^{2}} s\right)^{\frac{n}{2 \alpha}+1}$, where $B_{n}$ is the volume of the unit ball in $\mathbb{R}^{n}$.

Although analytic functions or harmonic functions satisfy the local submean value inequality, such an inequality is not available for $L^{(\alpha)}$-harmonic functions. Alternatively, we use the following estimate.

Lemma 1 Let $\gamma \in \mathbb{N}_{0}^{n}, k \in \mathbb{N}_{0}$ and $0<\delta_{0}<1$. Then, there exists a constant $C=$ $C\left(n, \alpha, \gamma, k, \delta_{0}\right)>0$ such that

$$
\left|R_{\alpha}^{\gamma, k}(X, Y)\right| \leq \frac{C}{S^{\frac{n}{2 \alpha}+1}} \int_{S_{\delta}^{(\alpha)}(Y)} \frac{r^{\frac{|\gamma|}{2 \alpha}+k}}{\left(t+r+|x-z|^{2 \alpha}\right)^{\frac{n+|\gamma|}{2 \alpha}+k+1}} d V(z, r)
$$

and

$$
\left|R_{\alpha}^{\gamma, k}(X, Y)\right| \leq \frac{C s^{\frac{|\gamma|}{2 \alpha}+k}}{t^{\frac{n+|\gamma|}{2 \alpha}+k+1}} \int_{S_{\delta}^{(\alpha)}(X)} \frac{r^{\frac{|\gamma|}{2 \alpha}+k}}{\left(s+r+|y-z|^{2 \alpha}\right)^{\frac{n+|\gamma|}{2 \alpha}+k+1}} d V(z, r)
$$

for all $X=(x, t), Y=(y, s) \in H$, and for all $\delta$ with $\delta_{0} \leq \delta<1$.

Proof We only prove Eq. 5, because the proof of Eq. 6 is similar. By Eq. 4 and the definition of $S_{\delta}^{(\alpha)}(Y)$, there exists a constant $C=C\left(n, \alpha, \gamma, k, \delta_{0}\right)>0$ such that for all $X=(x, t), Y=(y, s) \in H$ and $\delta_{0} \leq \delta<1$,

$$
\begin{aligned}
& S^{\frac{n}{2 \alpha}+1}\left|R_{\alpha}^{\gamma, k}(X, Y)\right| \\
& \quad \leq C s^{\frac{n}{2 \alpha}+1} \frac{s^{\frac{|\gamma|}{2 \alpha}+k}}{\left(t+s+|x-y|^{2 \alpha}\right)^{\frac{n+|\gamma|}{2 \alpha}+k+1}} \int_{S_{\delta}^{(\alpha)}(Y)} r^{\frac{|\gamma|}{2 \alpha}+k} d V(z, r)\left(\int_{S_{\delta}^{(\alpha)}(Y)} r^{\frac{|\gamma|}{2 \alpha}+k} d V(z, r)\right)^{-1}
\end{aligned}
$$




$$
\begin{aligned}
= & C \frac{s^{\frac{n+|\gamma|}{2 \alpha}+k+1}}{\left(t+s+|x-y|^{2 \alpha}\right)^{\frac{n+|\gamma|}{2 \alpha}+k+1}} \int_{S_{\delta}^{(\alpha)}(Y)} r^{\frac{|\gamma|}{2 \alpha}+k} d V(z, r) \\
& \times B_{n}^{-1}\left(\frac{1-\delta^{2}}{2 \delta s}\right)^{\frac{n}{2 \alpha}}\left(\frac{|\gamma|}{2 \alpha}+k+1\right)\left(\frac{1-\delta^{2}}{s}\right)^{\frac{\mid \gamma \gamma}{2 \alpha}+k+1} \\
& \times \frac{1}{(1+\delta)^{2\left(\frac{|\gamma|}{2 \alpha}+k+1\right)}-(1-\delta)^{2\left(\frac{|\gamma|}{2 \alpha}+k+1\right)}} \\
\leq & \frac{C\left(1-\delta^{2}\right)^{\frac{n+|\gamma|}{2 \alpha}}+k+1}{\left(t+s+|x-y|^{2 \alpha}\right)^{\frac{n+|\gamma|}{2 \alpha}}+k+1} \int_{S_{\delta}^{(\alpha)}(Y)} r^{\frac{|\gamma|}{2 \alpha}+k} d V(z, r) .
\end{aligned}
$$

Moreover, if $Z=(z, r) \in S_{\delta}^{(\alpha)}(Y)$, then we have

$$
\begin{aligned}
t+ & +|x-z|^{2 \alpha} \leq t+r+\{|x-y|+|y-z|\}^{2 \alpha} \\
& \leq t+\frac{1+\delta}{1-\delta} s+\left\{|x-y|+\left(\frac{2 \delta}{1-\delta^{2}} s\right)^{\frac{1}{2 \alpha}}\right\}^{2 \alpha} \\
& \leq t+\left(\frac{1+\delta}{1-\delta}+2^{2 \alpha} \frac{2 \delta}{1-\delta^{2}}\right) s+2^{2 \alpha}|x-y|^{2 \alpha} \\
& \leq \frac{12}{1-\delta^{2}}\left(t+s+|x-y|^{2 \alpha}\right) .
\end{aligned}
$$

Hence, we obtain

$$
s^{\frac{n}{2 \alpha}+1}\left|R_{\alpha}^{\gamma, k}(X, Y)\right| \leq C \int_{S_{\delta}^{(\alpha)}(Y)} \frac{r^{\frac{|\gamma|}{2 \alpha}+k}}{\left(t+r+|x-z|^{2 \alpha}\right)^{\frac{n+|\gamma|}{2 \alpha}+k+1}} d V(z, r) .
$$

The following lemma is Lemma 5 of [9]. We use this frequently in our later arguments.

Lemma 2 ( $\left[9\right.$, Lemma 5]) Let $\theta, \eta \in \mathbb{R}$. If $0<1+\theta<\eta-\frac{n}{2 \alpha}$, then there exists $a$ constant $C>0$ such that

$$
\int_{H} \frac{t^{\theta}}{\left(t+s+|x-y|^{2 \alpha}\right)^{\eta}} d V(x, t) \leq C s^{\theta-\eta+\frac{n}{2 \alpha}+1}
$$

for all $(y, s) \in H$.

\section{Separated Sequences}

Let $\mathbb{X}=\left\{X_{j}\right\}=\left\{\left(x_{j}, t_{j}\right)\right\}$ be a sequence in $H$ and $0<\delta<1$. We say that $\left\{X_{j}\right\}$ is $\delta$-separated in the $\alpha$-parabolic sense if $\alpha$-parabolic cylinders $S_{\delta}^{(\alpha)}\left(X_{j}\right)$ are pairwise disjoint. For $1 \leq p<\infty, \gamma \in \mathbb{N}_{0}^{n}, k \in \mathbb{N}_{0}$, and $u \in \boldsymbol{b}_{\alpha}^{p}$, define a sequence of real numbers $T_{p}^{\gamma, k} u$ by

$$
T_{p}^{\gamma, k} u=T_{p, \mathbb{X}}^{\gamma, k} u:=\left\{t_{j}^{\left(\frac{n}{2 \alpha}+1\right) \frac{1}{p}+\frac{|\gamma|}{2 \alpha}+k} \partial_{x}^{\gamma} \partial_{t}^{k} u\left(X_{j}\right)\right\} .
$$


We say that $\left\{X_{j}\right\}$ is a $\boldsymbol{b}_{\alpha}^{p}$-interpolating sequence of order $(\gamma, k)$ if $T_{p}^{\gamma, k}: \boldsymbol{b}_{\alpha}^{p} \rightarrow \ell^{p}$ is bounded and onto. Here we remark that if $\left\{X_{j}\right\}$ is a finite set, then $\ell^{p}$ consists of finite sequences.

In this section, we discuss a relation between separated sequences and boundedness of $T_{p}^{\gamma, k}$. We begin with recalling a main theorem of [10].

Lemma 3 ([10, Theorem 2]) Let $\mu$ be a $\sigma$-finite positive Borel measure on $H, 1 \leq p<$ $\infty, \gamma \in \mathbb{N}_{0}^{n}$ and $k \in \mathbb{N}_{0}$. Then, there exists a constant $C_{1}>0$ such that

$$
\int_{H}\left|\partial_{x}^{\gamma} \partial_{t}^{k} u\right|^{p} d \mu \leq C_{1} \int_{H}|u|^{p} d V
$$

for all $u \in \boldsymbol{b}_{\alpha}^{p}$ if and only if there exists a constant $C_{2}>0$ such that

$$
\mu\left(Q^{(\alpha)}(y, s)\right) \leq C_{2} s^{\frac{n}{2 \alpha}+1+\left(\frac{|\gamma|}{2 \alpha}+k\right) p}
$$

for all $(y, s) \in H$, where $Q^{(\alpha)}(y, s)$ is an $\alpha$-parabolic Carleson box defined by

$$
Q^{(\alpha)}(y, s):=\left\{(x, t) \in H ;\left|x_{\ell}-y_{\ell}\right|<2^{-1} s^{1 / 2 \alpha}(1 \leq \ell \leq n), s \leq t \leq 2 s\right\} .
$$

The following lemma shows that $S_{\delta}^{(\alpha)}(y, s)$ is nearly $Q^{(\alpha)}(y, s)$.

Lemma 4 For any $0<\delta<1$, there exist positive integers $M_{1}, M_{2}$ and constants $C_{1}, C_{2}$ with the following properties:

(1) For each $Y=(y, s) \in H$, there exist $Y_{i}=\left(y_{v_{i}}, s_{v_{i}}\right) \in H \quad\left(i=1,2, \cdots, M_{1}\right)$ such that

$$
Q^{(\alpha)}(Y) \subset \bigcup_{i=1}^{M_{1}} S_{\delta}^{(\alpha)}\left(Y_{i}\right) \text { and } s \leq s_{\nu_{i}} \leq C_{1} s .
$$

(2) For each $Y=(y, s) \in H$, there exist $Y_{i}=\left(y_{v_{i}}, s_{v_{i}}\right) \in H \quad\left(i=1,2, \cdots, M_{2}\right)$ such that

$$
S_{\delta}^{(\alpha)}(Y) \subset \bigcup_{i=1}^{M_{2}} Q^{(\alpha)}\left(Y_{i}\right) \text { and } \frac{1}{C_{2}} s \leq s_{v_{i}} \leq C_{2} s .
$$

Proof (1) For any $0<\delta<1$, put $a=\left(\frac{2 \delta}{1-\delta^{2}}\right)^{1 / 2 \alpha}$ and $b=\frac{1+\delta}{1-\delta}$. We may assume that $Y=(0, s)$. Let $K$ be the smallest positive integer satisfying $\frac{1}{2}<(K+1) a$. For a multiindex $v=\left(v_{1}, \ldots, v_{n}\right) \in \mathbb{Z}^{n}$, we put $y_{v}=\left(a s^{1 / 2 \alpha} v_{1}, \ldots, a s^{1 / 2 \alpha} v_{n}\right) \in \mathbb{R}^{n}$. Then,

$\left\{x \in \mathbb{R}^{n} ;\left|x_{\ell}\right|<2^{-1} s^{1 / 2 \alpha}(1 \leq \ell \leq n)\right\} \subset \bigcup\left\{x \in \mathbb{R}^{n} ;\left|x-y_{\nu}\right|<a s^{1 / 2 \alpha},\left|v_{\ell}\right| \leq K(1 \leq \ell \leq n)\right\}$.

Moreover, let $L$ be the smallest positive integer satisfying $2<b^{L+1}$, and let $s_{\lambda}=b^{\lambda} s$ for $\lambda=0,1, \ldots, L$, then

$$
[s, 2 s] \subset \bigcup\left\{\left[\frac{1-\delta}{1+\delta} s_{\lambda}, \frac{1+\delta}{1-\delta} s_{\lambda}\right] ; \lambda=0,1, \ldots, L\right\} \text { and } s \leq s_{\lambda} \leq b^{L} s .
$$

These show

$$
Q^{(\alpha)}(Y) \subset \bigcup\left\{S_{\delta}^{(\alpha)}\left(y_{\nu}, s_{\lambda}\right) ;\left|v_{\ell}\right| \leq K(1 \leq \ell \leq n), \lambda=0,1, \ldots, L\right\},
$$


and the integer $M_{1}=(2 K+1)^{n}(L+1)$ and the constant $C_{1}=b^{L}$ depend only on $n$, $\alpha$, and $\delta$. The proof of (2) is similar to that of (1).

By Lemmas 3 and 4, we have the following corollary.

Corollary 1 Let $\mu$ be a $\sigma$-finite positive Borel measure on $H, 1 \leq p<\infty, \gamma \in \mathbb{N}_{0}^{n}$ and $k \in \mathbb{N}_{0}$. Then, the following statements are equivalent:

(1) There exists a constant $C_{1}>0$ such that

$$
\int_{H}\left|\partial_{x}^{\gamma} \partial_{t}^{k} u\right|^{p} d \mu \leq C_{1} \int_{H}|u|^{p} d V
$$

for all $u \in \boldsymbol{b}_{\alpha}^{p}$

(2) There exists a constant $C_{2}>0$ such that

$$
\mu\left(Q^{(\alpha)}(y, s)\right) \leq C_{2} s^{\frac{n}{2 \alpha}+1+\left(\frac{|\gamma|}{2 \alpha}+k\right) p}
$$

for all $(y, s) \in H$;

(3) For any (or some) $0<\delta<1$, there exists a constant $C_{3}>0$ such that

$$
\mu\left(S_{\delta}^{(\alpha)}(y, s)\right) \leq C_{3} s^{\frac{n}{2 \alpha}+1+\left(\frac{|\gamma|}{2 \alpha}+k\right) p}
$$

for all $(y, s) \in H$.

For any finite set $E \subset H$, we denote by $\# E$ the number of points in $E$. The boundedness of $T_{p}^{\gamma, k}: b_{\alpha}^{p} \rightarrow \ell^{p}$ is characterized by Theorem 1 below. It follows from the proof of (2) it holds for all $\varepsilon$. Analogously, observing the proofs of (4) $\Longrightarrow(2)$ and (3) $\Longrightarrow(4)$, we see that if the statement (4) holds for some $\delta$ then it holds for all $\delta$.

Theorem 1 Let $1 \leq p<\infty, \gamma \in \mathbb{N}_{0}^{n}, k \in \mathbb{N}_{0}$ and $\mathbb{X}=\left\{X_{j}\right\}=\left\{\left(x_{j}, t_{j}\right)\right\}$ be a sequence in $H$. Then, the following conditions are equivalent:

(1) The operator $T_{p}^{\gamma, k}=T_{p, \mathbb{X}}^{\gamma, k}: \boldsymbol{b}_{\alpha}^{p} \rightarrow \ell^{p}$ is bounded;

(2) There exists a positive integer $K$ such that $\#\left(\mathbb{X} \cap Q^{(\alpha)}(Y)\right) \leq K$ for all $Y \in H$;

(3) For any (or some) $0<\varepsilon<1$, there exists a positive integer $L$ such that $\#(\mathbb{X} \cap$ $\left.S_{\varepsilon}^{(\alpha)}(Y)\right) \leq L$ for all $Y \in H$;

(4) For any (or some) $0<\delta<1, \mathbb{X}$ is a finite union of $\delta$-separated sequences, i.e., there exists a positive integer $M$ such that $\mathbb{X}=\mathbb{X}_{1} \cup \cdots \cup \mathbb{X}_{M}$ and each sequence $\mathbb{X}_{i}$ is $\delta$-separated in the $\alpha$-parabolic sense.

Proof (1) $\Longleftrightarrow(2)$. Put

$$
\mu:=\sum_{j} t_{j}^{\frac{n}{2 \alpha}+1+\left(\frac{|\gamma|}{2 \alpha}+k\right) p} \delta_{X_{j}}
$$

where $\delta_{X_{j}}$ denotes a Dirac measure at the point $X_{j}$. Then, since

$$
\left\|T_{p}^{\gamma, k} u\right\|_{\ell^{p}}^{p}=\sum_{j} t_{j}^{\frac{n}{2 \alpha}+1+\left(\frac{|\gamma|}{2 \alpha}+k\right) p}\left|\partial_{x}^{\gamma} \partial_{t}^{k} u\left(X_{j}\right)\right|^{p}=\int_{H}\left|\partial_{x}^{\gamma} \partial_{t}^{k} u\right|^{p} d \mu,
$$


it suffices to observe that $\mu$ satisfies Eq. 9 if and only if (2) holds. Let $Y=(y, s) \in H$. Then

$$
\begin{aligned}
\mu\left(Q^{(\alpha)}(y, s)\right) & =\sum_{j} t_{j}^{\frac{n}{2 \alpha}+1+\left(\frac{|\gamma|}{2 \alpha}+k\right) p} \delta_{X_{j}}\left(Q^{(\alpha)}(y, s)\right) \\
& \approx s^{\frac{n}{2 \alpha}+1+\left(\frac{|\gamma|}{2 \alpha}+k\right) p} \#\left(\mathbb{X} \cap Q^{(\alpha)}(y, s)\right),
\end{aligned}
$$

because $s \leq t_{j} \leq 2 s$ for $X_{j}=\left(x_{i}, t_{j}\right) \in Q^{(\alpha)}(y, s)$. Here $A \approx B$ means $C^{-1} A \leq B \leq$ $C A$ for some constant $C \geq 1$. This shows that $\mu$ satisfies Eq. 9 if and only if \#( $\mathbb{X} \cap$ $\left.Q^{(\alpha)}(y, s)\right) \leq K$ for all $(y, s) \in H$.

$(2) \Longleftrightarrow(3)$ is an immediate consequence of Lemma 4 .

(4) $\Longrightarrow(2)$. It is sufficient to consider the case that $\mathbb{X}=\left\{X_{j}\right\}$ is $\delta$-separated in the $\alpha$-parabolic sense for some $0<\delta<1$. Fix $Y=(y, s)=\left(y_{1}, \ldots, y_{n}, s\right) \in H$. By the definition of $Q^{(\alpha)}(y, s)$, if $X_{j} \in Q^{(\alpha)}(y, s)$ then

$$
S_{\delta}^{(\alpha)}\left(X_{j}\right) \subset\left\{(z, r) \in H ;\left|z_{\ell}-y_{\ell}\right|<\frac{s^{\frac{1}{2 \alpha}}}{2}+\left(\frac{2 \delta}{1-\delta^{2}} 2 s\right)^{\frac{1}{2 \alpha}}(1 \leq \ell \leq n), \frac{1-\delta}{1+\delta} s<r<\frac{1+\delta}{1-\delta} 2 s\right\} .
$$

Therefore, we obtain

$$
\begin{aligned}
& V\left(\bigcup\left\{S_{\delta}^{(\alpha)}\left(X_{j}\right) ; X_{j} \in Q^{(\alpha)}(y, s)\right\}\right) \\
& \leq V\left(\left\{(z, r) \in H ;\left|z_{\ell}-y_{\ell}\right|<\frac{s^{\frac{1}{2 \alpha}}}{2}+\left(\frac{2 \delta}{1-\delta^{2}} 2 s\right)^{\frac{1}{2 \alpha}}(1 \leq \ell \leq n),\right.\right. \\
& \left.\left.\quad \frac{1-\delta}{1+\delta} s<r<\frac{1+\delta}{1-\delta} 2 s\right\}\right) \\
& =\left\{1+2\left(\frac{4 \delta}{1-\delta^{2}}\right)^{\frac{1}{2 \alpha}}\right\}^{n} \frac{1+6 \delta+\delta^{2}}{1-\delta^{2}} s^{\frac{n}{2 \alpha}+1}=C s^{\frac{n}{2 \alpha}+1} .
\end{aligned}
$$

Moreover, for each $X \in Q^{(\alpha)}(y, s)$, we have

$$
V\left(S_{\delta}^{(\alpha)}(X)\right) \geq V\left(S_{\delta}^{(\alpha)}(0, s)\right)=2 B_{n}\left(\frac{2 \delta}{1-\delta^{2}}\right)^{\frac{n}{2 \alpha}+1} s^{\frac{n}{2 \alpha}+1},
$$

where $B_{n}$ is the volume of the unit ball in $\mathbb{R}^{n}$. Hence, if we take an integer $K=$ $K(n, \alpha, \delta)$ satisfying

$$
V\left(\cup\left\{S_{\delta}^{(\alpha)}\left(X_{j}\right) ; X_{j} \in Q^{(\alpha)}(y, s)\right\}\right) \leq K V\left(S_{\delta}^{(\alpha)}(0, s)\right),
$$

then at most $K$ of the points $X_{j}$ belong to $Q^{(\alpha)}(y, s)$, because $S_{\delta}^{(\alpha)}\left(X_{j}\right)$ are pairwise disjoint.

(3) $\Longrightarrow$ (4). Let $0<\delta<1$ be arbitrary. First, we show that there exists $0<\varepsilon<1$ such that for each $Y \in H, S_{\delta}^{(\alpha)}(Y) \cap S_{\delta}^{(\alpha)}(Z)=\emptyset$ whenever $Z \notin S_{\varepsilon}^{(\alpha)}(Y)$. In fact, let $Y=(y, s) \in H$ be fixed and choose a constant $\rho$ such that $\delta<\rho<1$ and $\frac{1-\rho}{1+\rho}<$ $\left(\frac{1-\delta}{1+\delta}\right)^{2}$. If $Z=(z, r) \in H$ and $r \leq \frac{1-\rho}{1+\rho} s$, then $S_{\delta}^{(\alpha)}(y, s) \cap S_{\delta}^{(\alpha)}(z, r)=\emptyset$, because

$$
\sup \left\{t ;(x, t) \in S_{\delta}^{(\alpha)}(z, r)\right\}=\frac{1+\delta}{1-\delta} r<\frac{1-\delta}{1+\delta} s=\inf \left\{t ;(x, t) \in S_{\delta}^{(\alpha)}(y, s)\right\} .
$$


Similarly, if $\frac{1+\rho}{1-\rho} s \leq r$ then $S_{\delta}^{(\alpha)}(y, s) \cap S_{\delta}^{(\alpha)}(z, r)=\emptyset$. Hence, $S_{\delta}^{(\alpha)}(y, s) \cap S_{\delta}^{(\alpha)}(z, r)=$ $\emptyset$ whenever $r \notin\left(\frac{1-\rho}{1+\rho} s, \frac{1+\rho}{1-\rho} s\right)$. Moreover, if we take a constant $\varepsilon$ such that $\rho<$ $\varepsilon<1$ and $\left(\frac{2 \delta}{1-\delta^{2}} \frac{1+\rho}{1-\rho}\right)^{1 / 2 \alpha}+\left(\frac{2 \delta}{1-\delta^{2}}\right)^{1 / 2 \alpha}<\left(\frac{2 \varepsilon}{1-\varepsilon^{2}}\right)^{1 / 2 \alpha}$, then $(z, r) \notin S_{\varepsilon}^{(\alpha)}(y, s)$ implies $r \notin$ $\left(\frac{1-\rho}{1+\rho} s, \frac{1+\rho}{1-\rho} s\right)$, so that $S_{\delta}^{(\alpha)}(y, s) \cap S_{\delta}^{(\alpha)}(z, r)=\emptyset$.

Next, take a positive integer $L$ such that $\#\left(\mathbb{X} \cap S_{\varepsilon}^{(\alpha)}(Y)\right) \leq L$ for all $Y \in H$, where $\varepsilon$ is the constant defined above. Then, for any $j, m \in \mathbb{N}, S_{\delta}^{(\alpha)}\left(X_{j}\right) \cap S_{\delta}^{(\alpha)}\left(X_{m}\right)=\emptyset$ holds whenever $X_{m} \notin S_{\varepsilon}^{(\alpha)}\left(X_{j}\right)$. Therefore, for each $j \in \mathbb{N}$ we obtain

$$
\#\left(\left\{m \in \mathbb{N} ; m \neq j, S_{\delta}^{(\alpha)}\left(X_{j}\right) \cap S_{\delta}^{(\alpha)}\left(X_{m}\right) \neq \emptyset\right\}\right) \leq L-1 .
$$

Now we will choose a subsequence $\mathbb{X}_{1} \subset \mathbb{X}=\left\{X_{j}\right\}_{j \in \mathbb{N}}$ such that $\mathbb{X}_{1}$ is $\delta$-separated in the $\alpha$-parabolic sense. Put

$$
L_{1}:=\max _{j \in \mathbb{N}} \#\left(\left\{m \in \mathbb{N} ; m \neq j, S_{\delta}^{(\alpha)}\left(X_{j}\right) \cap S_{\delta}^{(\alpha)}\left(X_{m}\right) \neq \emptyset\right\}\right) \quad(\leq L-1),
$$

and let $j(1)$ be a first integer for which the maximum in Eq. 11 is attained. If

$$
L_{1}=\max _{j \in \mathbb{N} \backslash\{j(1)\}} \#\left(\left\{m \in \mathbb{N} \backslash\{j(1)\} ; m \neq j, S_{\delta}^{(\alpha)}\left(X_{j}\right) \cap S_{\delta}^{(\alpha)}\left(X_{m}\right) \neq \emptyset\right\}\right)
$$

also holds, then let $j(2)$ be a first integer in $\mathbb{N} \backslash\{j(1)\}$ for which the maximum in Eq. 12 is attained. Continuing in this fashion if it is possible, we can choose a subsequence $\mathbb{X}_{1}=\left\{X_{j(k)}\right\}_{k}$ (the sequence $\mathbb{X}_{1}$ is finite or infinite) such that

$$
L_{1}=\#\left(\left\{m \in \mathbb{N} \backslash\{j(1), \ldots, j(k-1)\} ; m \neq j(k), S_{\delta}^{(\alpha)}\left(X_{j(k)}\right) \cap S_{\delta}^{(\alpha)}\left(X_{m}\right) \neq \emptyset\right\}\right)
$$

for all $k \geq 1$ and

$$
L_{1}-1 \geq \max _{j \in \mathbb{N} \backslash\{j(k)\}_{k}} \#\left(\left\{m \in \mathbb{N} \backslash\{j(k)\}_{k} ; m \neq j, S_{\delta}^{(\alpha)}\left(X_{j}\right) \cap S_{\delta}^{(\alpha)}\left(X_{m}\right) \neq \emptyset\right\}\right) .
$$

We claim that $\mathbb{X}_{1}=\left\{X_{j(k)}\right\}_{k}$ is $\delta$-separated in the $\alpha$-parabolic sense. In fact, suppose contrarily that there exist $X_{j(k)}, X_{j(\ell)} \in \mathbb{X}_{1}$ with $j(k)>j(\ell)$ such that $S_{\delta}^{(\alpha)}\left(X_{j(k)}\right) \cap$ $S_{\delta}^{(\alpha)}\left(X_{j(\ell)}\right) \neq \emptyset$. Then, by Eq. 13, we have

$$
L_{1}+1 \leq \#\left(\left\{m \in \mathbb{N} ; m \neq j(k), S_{\delta}^{(\alpha)}\left(X_{j(k)}\right) \cap S_{\delta}^{(\alpha)}\left(X_{m}\right) \neq \emptyset\right\}\right),
$$

because $j(\ell) \in\{j(1), \ldots, j(k-1)\}$. This contradicts Eq. 11, and hence $\mathbb{X}_{1}$ is $\delta$ separated in the $\alpha$-parabolic sense.

We repeat the similar argument to $\mathbb{X} \backslash \mathbb{X}_{1}$, and choose a subsequence $\mathbb{X}_{2} \subset \mathbb{X} \backslash \mathbb{X}_{1}$ which is also $\delta$-separated in the $\alpha$-parabolic sense. Because of Eq. 14 this operation stops in finite steps, so that we have $\mathbb{X}=\mathbb{X}_{1} \cup \cdots \cup \mathbb{X}_{M}$, and each sequence $\mathbb{X}_{i}$ is $\delta$-separated in the $\alpha$-parabolic sense.

Theorem 1 gives us the following useful consequence.

Corollary 2 If a sequence $\mathbb{X}$ is $\delta$-separated in the $\alpha$-parabolic sense for some $0<\delta<1$, then for any $0<\eta<1, \mathbb{X}$ consists of a finite union of $\eta$-separated sequences in the $\alpha$-parabolic sense. 


\section{Interpolating Sequences of $b_{\alpha}^{p}$}

In this section, we shall state our $\boldsymbol{b}_{\alpha}^{p}$-interpolation theorem. Let $1 \leq p<\infty, \gamma \in \mathbb{N}_{0}^{n}$, $k \in \mathbb{N}_{0}$ and $\mathbb{X}=\left\{X_{j}\right\}=\left\{\left(x_{j}, t_{j}\right)\right\}$ be a sequence in $H$. For $\left\{\lambda_{j}\right\} \in \ell^{p}$, let

$$
\left(U_{p}^{\gamma, k}\left\{\lambda_{j}\right\}\right)(X)=\left(U_{p, \mathbb{X}}^{\gamma, k}\left\{\lambda_{j}\right\}\right)(X)=\sum_{j} \lambda_{j} t_{j}^{\left(\frac{n}{2 \alpha}+1\right)\left(1-\frac{1}{p}\right)} R_{\alpha}^{\gamma, k}\left(X, X_{j}\right) \quad(X \in H) .
$$

The following lemma shows that the operator $U_{p}^{\gamma, k}: \ell^{p} \rightarrow \boldsymbol{b}_{\alpha}^{p}$ is bounded whenever $\left\{X_{j}\right\}$ is $\delta$-separated in the $\alpha$-parabolic sense.

Lemma 5 Suppose $\mathbb{X}=\left\{X_{j}\right\}=\left\{\left(x_{j}, t_{j}\right)\right\}$ is $\delta$-separated in the $\alpha$-parabolic sense. Then, for $1<p<\infty$, the operator $U_{p}^{\gamma, k}=U_{p, \mathbb{X}}^{\gamma, k}: \ell^{p} \rightarrow \boldsymbol{b}_{\alpha}^{p}$ is bounded for each $(\gamma, k) \in$ $\mathbb{N}_{0}^{n} \times \mathbb{N}_{0}$, while the operator $U_{1}^{\gamma, k}=U_{1, \mathbb{X}}^{\gamma, k}: \ell^{1} \rightarrow \boldsymbol{b}_{\alpha}^{1}$ is bounded for each $(\gamma, k) \in \mathbb{N}_{0}^{n} \times$ $\mathbb{N}_{0} \backslash\{(0,0)\}$.

Proof Let $\left\{\lambda_{j}\right\} \in \ell^{p}, \gamma \in \mathbb{N}_{0}^{n}$ and $k \in \mathbb{N}_{0}$. Then, by Eq. 5, we have

$$
\begin{aligned}
& \left|\sum_{j} \lambda_{j} t_{j}^{\left(\frac{n}{2 \alpha}+1\right)\left(1-\frac{1}{p}\right)} R_{\alpha}^{\gamma, k}\left(X, X_{j}\right)\right| \\
& \quad \leq C \sum_{j}\left|\lambda_{j}\right| t_{j}^{-\left(\frac{n}{2 \alpha}+1\right) \frac{1}{p}} \int_{S_{\delta}^{(\alpha)}\left(X_{j}\right)} \frac{r^{\frac{|\gamma|}{2 \alpha}+k}}{\left(t+r+|x-z|^{2 \alpha}\right)^{\frac{n+|\gamma|}{2 \alpha}+k+1}} d V(z, r) \\
& \quad=C \int_{H} f(z, r) \frac{r^{\frac{|\gamma|}{2 \alpha}+k}}{\left(t+r+|x-z|^{2 \alpha}\right)^{\frac{n+|\gamma|}{2 \alpha}+k+1}} d V(z, r),
\end{aligned}
$$

where

$$
f(z, r):=\sum_{j}\left|\lambda_{j}\right| t_{j}^{-\left(\frac{n}{2 \alpha}+1\right) \frac{1}{p}} \chi_{j}(z, r)
$$

and $\chi_{j}$ denotes the characteristic function of $S_{\delta}^{(\alpha)}\left(X_{j}\right)$.

Let $1<p<\infty$ and $q$ be the exponent conjugate to $p$. Then the Hölder inequality and Lemma 2 imply that

$$
\begin{aligned}
& \left|\sum_{j} \lambda_{j} t_{j}^{\left(\frac{n}{2 \alpha}+1\right)\left(1-\frac{1}{p}\right)} R_{\alpha}^{\gamma, k}\left(X, X_{j}\right)\right|^{p} \\
& \quad \leq C\left(\int_{H} \frac{f(z, r)}{\left(t+r+|x-z|^{2 \alpha}\right)^{\frac{n}{2 \alpha}+1}} d V(z, r)\right)^{p} \\
& \quad \leq C \int_{H} \frac{f(z, r)^{p} r^{1 / q}}{\left(t+r+|x-z|^{2 \alpha}\right)^{\frac{n}{2 \alpha}+1}} d V(z, r)\left(\int_{H} \frac{r^{-1 / p}}{\left(t+r+|x-z|^{2 \alpha}\right)^{\frac{n}{2 \alpha}+1}} d V(z, r)\right)^{p / q} \\
& \quad \leq C t^{-1 / q} \int_{H} \frac{f(z, r)^{p} r^{1 / q}}{\left(t+r+|x-z|^{2 \alpha}\right)^{\frac{n}{2 \alpha}+1}} d V(z, r) .
\end{aligned}
$$


It follows from the Fubini theorem and Lemma 2 that

$$
\begin{aligned}
& \left\|\sum_{j} \lambda_{j} t_{j}^{\left(\frac{n}{2 \alpha}+1\right)\left(1-\frac{1}{p}\right)} R_{\alpha}^{\gamma, k}\left(\cdot, X_{j}\right)\right\|_{p}^{p} \\
& \quad \leq C \int_{H} f(z, r)^{p} r^{1 / q} \int_{H} \frac{t^{-1 / q}}{\left(t+r+|x-z|^{2 \alpha}\right)^{\frac{n}{2 \alpha}+1}} d V(x, t) d V(z, r) \\
& \quad \leq C \int_{H} f(z, r)^{p} d V(z, r) \\
& \quad=C \sum_{m} \int_{S_{\delta}^{(\alpha)}\left(X_{m}\right)}\left(\sum_{j}\left|\lambda_{j}\right| t_{j}^{-\left(\frac{n}{2 \alpha}+1\right) \frac{1}{p}} \chi_{j}(z, r)\right)^{p} d V(z, r) \\
& \quad=C \sum_{m}\left|\lambda_{m}\right|^{p} t_{m}^{-\left(\frac{n}{2 \alpha}+1\right)} V\left(S_{\delta}^{(\alpha)}\left(X_{m}\right)\right) \\
& \quad \leq C \sum_{m}\left|\lambda_{m}\right|^{p} .
\end{aligned}
$$

Hence for any $(\gamma, k) \in \mathbb{N}_{0}^{n} \times \mathbb{N}_{0}$, the series in Eq. 15 converges in norm and the operator $U_{p}^{\gamma, k}: \ell^{p} \rightarrow L^{p}$ is bounded.

Next we assume that $p=1$ and $(\gamma, k) \neq(0,0)$. Then, the Fubini theorem and Lemma 2 again show that

$$
\begin{aligned}
& \left\|\sum_{j} \lambda_{j} R_{\alpha}^{\gamma, k}\left(\cdot, X_{j}\right)\right\|_{1} \\
& \quad \leq C \int_{H} f(z, r) r^{\frac{|\gamma|}{2 \alpha}+k} \int_{H} \frac{1}{\left(t+r+|x-z|^{2 \alpha}\right)^{\frac{n+|\gamma|}{2 \alpha}+k+1}} d V(x, t) d V(z, r) \\
& \quad \leq C \int_{H} f(z, r) d V(z, r) \\
& \quad \leq C \sum_{m}\left|\lambda_{m}\right| .
\end{aligned}
$$

This completes the proof.

Remark 1 We note that for any $\gamma^{\prime} \in \mathbb{N}_{0}^{n}$ is a multi-index and $\ell \in \mathbb{N}_{0}$. A series

$$
\sum_{j} \lambda_{j} t_{j}^{\left(\frac{n}{2 \alpha}+1\right)\left(1-\frac{1}{p}\right)} \partial_{x}^{\gamma^{\prime}} \partial_{t}^{\ell} R_{\alpha}^{\gamma, k}\left(X, X_{j}\right)
$$

converges uniformly on $\mathbb{R}^{n} \times[\tau, \infty)$ for every $\tau>0$. In fact, by Theorem 5.4 of [7], there exists a constant $C>0$ such that $\left|\partial_{x}^{\gamma^{\prime}} \partial_{t}^{\ell} u(x, t)\right| \leq C t^{-\left(\frac{\left|\gamma^{\prime}\right|}{2 \alpha}+\ell\right)-\left(\frac{n}{2 \alpha}+1\right) \frac{1}{p}}\|u\|_{p}$ for all $u \in \boldsymbol{b}_{\alpha}^{p}$ and $(x, t) \in H$. Hence, the norm convergence of the series Eq. 15 implies the uniform convergence of the series Eq. 16 on $\mathbb{R}^{n} \times[\tau, \infty)$ for every $\tau>0$. 
Lemma 6 Let $1<p<\infty, \frac{1}{p}+\frac{1}{q}=1, \gamma \in \mathbb{N}_{0}^{n}, k \in \mathbb{N}_{0}$ and $0<\delta_{0}<1$. If $\mathbb{X}=\left\{X_{j}\right\}=$ $\left\{\left(x_{j}, t_{j}\right)\right\}$ is $\delta$-separated in the $\alpha$-parabolic sense with $\delta_{0} \leq \delta<1$, then there exists a constant $C=C\left(n, \alpha, p, \gamma, k, \delta_{0}\right)>0$ such that

$$
\left|\sum_{j} \lambda_{j} t_{j}^{\left(\frac{n}{2 \alpha}+1\right) \frac{1}{q}+\theta} \partial_{x}^{\gamma} \partial_{t}^{k} R_{\alpha}\left(X, X_{j}\right)\right|^{p} \leq C t^{-\frac{1}{q}-\left(\frac{|\gamma|}{2 \alpha}+k-\theta\right) p} \sum_{j} \frac{\left|\lambda_{j}\right|^{p} t_{j}^{\frac{1}{q}}}{\left(t+t_{j}+\left|x-x_{j}\right|^{2 \alpha}\right)^{\frac{n}{2 \alpha}+1}}
$$

for all $\left\{\lambda_{j}\right\} \in \ell^{p}, 0<\theta \leq \frac{|\gamma|}{2 \alpha}+k$, and $X=(x, t) \in H$.

Proof By Eq. 4, we have

$$
\begin{aligned}
& \left|\sum_{j} \lambda_{j} t_{j}^{\left(\frac{n}{2 \alpha}+1\right) \frac{1}{q}+\theta} \partial_{x}^{\gamma} \partial_{t}^{k} R_{\alpha}\left(X, X_{j}\right)\right| \\
& \leq C \sum_{j} \frac{\left|\lambda_{j}\right| t_{j}^{\left(\frac{n}{2 \alpha}+1\right) \frac{1}{q}+\theta}}{\left(t+t_{j}+\left|x-x_{j}\right|^{2 \alpha}\right)^{\frac{n+|\gamma|}{2 \alpha}+k+1}} \\
& \leq C t^{-\left(\frac{|\gamma|}{2 \alpha}+k-\theta\right) \sum_{j}\left|\lambda_{j}\right| t_{j}^{\left(\frac{n}{2 \alpha}+1\right) \frac{1}{q}}} b_{\alpha}\left(X, X_{j}\right),
\end{aligned}
$$

where $b_{\alpha}\left(X, X_{j}\right)=\left(t+t_{j}+\left|x-x_{j}\right|^{2 \alpha}\right)^{-\left(\frac{n}{2 \alpha}+1\right)}$. Therefore, the Hölder inequality implies that

$$
\begin{aligned}
& \left|\sum_{j} \lambda_{j} t_{j}^{\left(\frac{n}{2 \alpha}+1\right) \frac{1}{q}+\theta} \partial_{x}^{\gamma} \partial_{t}^{k} R_{\alpha}\left(X, X_{j}\right)\right|^{p} \\
& \quad \leq C^{p} t^{-\left(\frac{|\gamma|}{2 \alpha}+k-\theta\right) p}\left(\sum_{j}\left|\lambda_{j}\right| t_{j}^{\frac{1}{p q}} b_{\alpha}\left(X, X_{j}\right)^{\frac{1}{p}} \cdot t_{j}^{\left(\frac{n}{2 \alpha}+1\right) \frac{1}{q}} t_{j}^{-\frac{1}{p q}} b_{\alpha}\left(X, X_{j}\right)^{\frac{1}{q}}\right)^{p} \\
& \quad \leq C^{p} t^{-\left(\frac{|\gamma|}{2 \alpha}+k-\theta\right) p} \sum_{j}\left|\lambda_{j}\right|^{p} t_{j}^{\frac{1}{q}} b_{\alpha}\left(X, X_{j}\right)\left(\sum_{j} t_{j}^{\frac{n}{2 \alpha}+1-\frac{1}{p}} b_{\alpha}\left(X, X_{j}\right)\right)^{p / q} .
\end{aligned}
$$

Since $1 / 3 \leq t_{j} / r \leq 3$ for $(z, r) \in S_{\delta / 2}^{(\alpha)}\left(X_{j}\right)$, the proof of Lemma 1 implies that

$$
\begin{aligned}
\sum_{j} t_{j}^{\frac{n}{2 \alpha}+1-\frac{1}{p}} b_{\alpha}\left(X, X_{j}\right) & \leq C \sum_{j} \int_{S_{\delta / 2}^{(\alpha)}\left(X_{j}\right)} \frac{r^{-\frac{1}{p}}}{\left(t+r+|x-z|^{2 \alpha}\right)^{\frac{n}{2 \alpha}+1}} d V(z, r) \\
& \leq C \int_{H} \frac{r^{-\frac{1}{p}}}{\left(t+r+|x-z|^{2 \alpha}\right)^{\frac{n}{2 \alpha}+1}} d V(z, r) \\
& \leq C t^{-\frac{1}{p}}
\end{aligned}
$$

where $C$ is independent of $\delta$.

The following $\boldsymbol{b}_{\alpha}^{p}$-interpolation theorem is the main result of this paper. 
Theorem 2 Let $1 \leq p<\infty, \gamma \in \mathbb{N}_{0}^{n}$ and $k \in \mathbb{N}_{0}$. Then, there exists a positive number $\delta_{0}$ such that if $\left\{X_{j}\right\}$ is $\delta$-separated in the $\alpha$-parabolic sense with $\delta \geq \delta_{0}$ then $\left\{X_{j}\right\}$ is a $\boldsymbol{b}_{\alpha}^{p}$-interpolating sequence of order $(\gamma, k)$.

Proof First, we note that $s^{\frac{n+2|\gamma|}{2 \alpha}+k+1} \partial_{x}^{2 \gamma} \partial_{t}^{k} R_{\alpha}(y, s ; y, s)$ is constant on $H$. In fact, by Eq. 3 and the definition of $R_{\alpha}^{\gamma, k}$, we see

$$
\partial_{x}^{2 \gamma} \partial_{t}^{k} R_{\alpha}(y, s ; y, s)=-2^{-\left(\frac{n+2|\gamma|}{2 \alpha}+k\right)} s^{-\left(\frac{n+2|\gamma|}{2 \alpha}+k+1\right)} \partial_{x}^{2 \gamma} \partial_{t}^{k+1} W^{(\alpha)}(0,1) .
$$

Put $d_{\gamma, k}=s^{\frac{n+2|\gamma|}{2 \alpha}+k+1} \partial_{x}^{2 \gamma} \partial_{t}^{k} R_{\alpha}(y, s ; y, s)$. By the proof of (2) of Proposition 1 of [10], we have $\partial_{x}^{2 \gamma} \partial_{t}^{k+1} W^{(\alpha)}(0,1) \neq 0$, and so is $d_{\gamma, k}$.

Suppose that $\left\{X_{j}\right\}=\left\{\left(x_{j}, t_{j}\right)\right\}$ is $\delta$-separated in the $\alpha$-parabolic sense. Let $1<p<$ $\infty$ and $q$ be the exponent conjugate to $p$. Then, by Lemma 5, the operator $U_{p}^{\gamma, 0}$ : $\ell^{p} \rightarrow \boldsymbol{b}_{\alpha}^{p}$ is bounded, and thus $T_{p}^{\gamma, k} U_{p}^{\gamma, 0}: \ell^{p} \rightarrow \ell^{p}$ is also bounded by Theorem 1. We show that there exists a positive number $\delta_{0}$ such that if $\delta \geq \delta_{0}$ then $\| T_{p}^{\gamma, k} U_{p}^{\gamma, 0}-$ $d_{\gamma, k} I \|<\left|d_{\gamma, k}\right|$, where $I$ is the identity operator on $\ell^{p}$. In fact, the operator $T_{p}^{\gamma, k} U_{p}^{\gamma, 0}-$ $d_{\gamma, k} I$ maps a sequence $\left\{\lambda_{j}\right\}$ in $\ell^{p}$ to a sequence $\left\{\xi_{m}\right\}$ in $\ell^{p}$ given by

$$
\xi_{m}=t_{m}^{\left(\frac{n}{2 \alpha}+1\right) \frac{1}{p}+\frac{|\gamma|}{2 \alpha}+k}\left(\partial_{x}^{\gamma} \partial_{t}^{k} U_{p}^{\gamma, 0}\left\{\lambda_{j}\right\}\right)\left(X_{m}\right)-d_{\gamma, k} \lambda_{m}
$$

From Remark 1, we may differentiate term by term, so that

$$
\begin{aligned}
\xi_{m} & =t_{m}^{\left(\frac{n}{2 \alpha}+1\right) \frac{1}{p}+\frac{|\gamma|}{2 \alpha}+k} \sum_{j} \lambda_{j} t_{j}^{\left(\frac{n}{2 \alpha}+1\right) \frac{1}{q}} \partial_{x}^{\gamma} \partial_{t}^{k} R_{\alpha}^{\gamma, 0}\left(X_{m}, X_{j}\right)-d_{\gamma, k} \lambda_{m} \\
& =t_{m}^{\left(\frac{n}{2 \alpha}+1\right) \frac{1}{p}+\frac{|\gamma|}{2 \alpha}+k} \sum_{j \neq m} \lambda_{j} t_{j}^{\left(\frac{n}{2 \alpha}+1\right) \frac{1}{q}+\frac{|\gamma|}{2 \alpha}} \partial_{x}^{2 \gamma} \partial_{t}^{k} R_{\alpha}\left(X_{m}, X_{j}\right) .
\end{aligned}
$$

By Lemma 6, we obtain

$$
\begin{aligned}
\sum_{m}\left|\xi_{m}\right|^{p} & \leq \sum_{m} t_{m}^{\frac{n}{2 \alpha}+1+\left(\frac{|\gamma|}{2 \alpha}+k\right) p}\left|\sum_{j \neq m} \lambda_{j} t_{j}^{\left(\frac{n}{2 \alpha}+1\right) \frac{1}{q}+\frac{|\gamma|}{2 \alpha}} \partial_{x}^{2 \gamma} \partial_{t}^{k} R_{\alpha}\left(X_{m}, X_{j}\right)\right|^{p} \\
& \leq C \sum_{m} t_{m}^{\frac{n}{2 \alpha}+1+\left(\frac{|\gamma|}{2 \alpha}+k\right) p} t_{m}^{-\frac{1}{q}-\left(\frac{|\gamma|}{2 \alpha}+k\right) p} \sum_{j \neq m} \frac{\left|\lambda_{j}\right|^{p} t_{j}^{\frac{1}{q}}}{\left(t_{m}+t_{j}+\left|x_{m}-x_{j}\right|^{2 \alpha}\right)^{\frac{n}{2 \alpha}+1}} \\
& =C \sum_{j}\left|\lambda_{j}\right|^{p}\left(t_{j}^{\frac{1}{q}} \sum_{m \neq j} \frac{t_{m}^{\frac{n}{2 \alpha}+1-\frac{1}{q}}}{\left(t_{m}+t_{j}+\left|x_{m}-x_{j}\right|^{2 \alpha}\right)^{\frac{n}{2 \alpha}+1}}\right)
\end{aligned}
$$


Since $1 / 3 \leq t_{m} / r \leq 3$ for $(z, r) \in S_{\delta / 2}^{(\alpha)}\left(X_{m}\right)$, the proof of Lemma 1 implies that

$$
\begin{aligned}
& t_{j}^{\frac{1}{q}} \sum_{m \neq j} \frac{t_{m}^{\frac{n}{2 \alpha}+1-\frac{1}{q}}}{\left(t_{m}+t_{j}+\left|x_{m}-x_{j}\right|^{2 \alpha}\right)^{\frac{n}{2 \alpha}+1}} \\
& \quad \leq C t_{j}^{\frac{1}{q}} \sum_{m \neq j} \int_{S_{\delta / 2}^{(\alpha)}\left(X_{m}\right)} \frac{r^{-\frac{1}{q}}}{\left(r+t_{j}+\left|z-x_{j}\right|^{2 \alpha}\right)^{\frac{n}{2 \alpha}+1}} d V(z, r) \\
& \quad \leq C t_{j}^{\frac{1}{q}} \int_{H \backslash S_{\delta}^{(\alpha)}\left(X_{j}\right)} \frac{r^{-\frac{1}{q}}}{\left(r+t_{j}+\left|z-x_{j}\right|^{2 \alpha}\right)^{\frac{n}{2 \alpha}+1}} d V(z, r) \\
& \quad=C t_{j}^{\frac{1}{q}} \int_{H \backslash S_{\delta}^{(\alpha)}(0,1)} \frac{\left(t_{j} t\right)^{-\frac{1}{q}}}{\left(t_{j} t+t_{j}+\left|t_{j}^{\frac{1}{2 \alpha}} x+x_{j}-x_{j}\right|^{2 \alpha}\right)^{\frac{n}{2 \alpha}+1}} t_{j}^{\frac{n}{2 \alpha}+1} d V(x, t) \\
& \quad=C \int_{H \backslash S_{\delta}^{(\alpha)}(0,1)} \frac{t^{-\frac{1}{q}}}{\left(t+1+|x|^{2 \alpha}\right)^{\frac{n}{2 \alpha}+1}} d V(x, t) .
\end{aligned}
$$

Here we may assume that $C$ is independent of $\delta$, because our concern is only $\delta$ 's near 1. Therefore, we obtain

$$
\left\|T_{p}^{\gamma, k} U_{p}^{\gamma, 0}-d_{\gamma, k} I\right\|^{p} \leq C \int_{H \backslash S_{\delta}^{(\alpha)}(0,1)} \frac{t^{-\frac{1}{q}}}{\left(t+1+|x|^{2 \alpha}\right)^{\frac{n}{2 \alpha}+1}} d V(x, t) .
$$

Since the right-hand side of the inequality of Eq. 17 tends to 0 as $\delta \rightarrow 1$ by Lemma 2, there exists a positive number $\delta_{0}$ such that if $\delta \geq \delta_{0}$ then $\left\|T_{p}^{\gamma, k} U_{p}^{\gamma, 0}-d_{\gamma, k} I\right\|<\left|d_{\gamma, k}\right|$. Hence, if $\delta \geq \delta_{0}$, then $T_{p}^{\gamma, k} U_{p}^{\gamma, 0}$ is invertible on $\ell^{p}$ and $T_{p}^{\gamma, k}\left\{U_{p}^{\gamma, 0}\left(T_{p}^{\gamma, k} U_{p}^{\gamma, 0}\right)^{-1}\right\}=I$. Therefore $T_{p}^{\gamma, k}$ is onto.

Next we consider the case $p=1$. By Lemma 5 , the operator $U_{1}^{\gamma, 1}: \ell^{1} \rightarrow \boldsymbol{b}_{\alpha}^{1}$ is bounded, and thus $T_{1}^{\gamma, k} U_{1}^{\gamma, 1}: \ell^{1} \rightarrow \ell^{1}$ is also bounded by Theorem 1 . We show that there exists a positive number $\delta_{0}$ such that if $\delta \geq \delta_{0}$ then $\left\|T_{1}^{\gamma, k} U_{1}^{\gamma, 1}+2 d_{\gamma, k+1} I\right\|<$ $2\left|d_{\gamma, k+1}\right|$. The operator $T_{1}^{\gamma, k} U_{1}^{\gamma, 1}+2 d_{\gamma, k+1} I$ maps a sequence $\left\{\lambda_{j}\right\}$ in $\ell^{1}$ to a sequence $\left\{\xi_{m}\right\}$ in $\ell^{1}$ given by

$$
\begin{aligned}
\xi_{m} & =t_{m}^{\frac{n}{2 \alpha}+1+\frac{|\gamma|}{2 \alpha}+k}\left(\partial_{x}^{\gamma} \partial_{t}^{k} U_{1}^{\gamma, 1}\left\{\lambda_{j}\right\}\right)\left(X_{m}\right)+2 d_{\gamma, k+1} \lambda_{m} \\
& =t_{m}^{\frac{n}{2 \alpha}+1+\frac{|\gamma|}{2 \alpha}+k} \sum_{j} \lambda_{j} \partial_{x}^{\gamma} \partial_{t}^{k} R_{\alpha}^{\gamma, 1}\left(X_{m}, X_{j}\right)+2 d_{\gamma, k+1} \lambda_{m} \\
& =-2 t_{m}^{\frac{n}{2 \alpha}+1+\frac{|\gamma|}{2 \alpha}+k} \sum_{j \neq m} \lambda_{j} t_{j}^{\frac{|\gamma|}{2 \alpha}+1} \partial_{x}^{2 \gamma} \partial_{t}^{k+1} R_{\alpha}\left(X_{m}, X_{j}\right) .
\end{aligned}
$$


Similarly to the case $p>1$, we have

$$
\begin{aligned}
\sum_{m}\left|\xi_{m}\right| & \leq C \sum_{j}\left|\lambda_{j}\right| t_{j}^{\frac{|\gamma|}{2 \alpha}+1} \sum_{m \neq j} t_{m}^{\frac{n}{2 \alpha}+1+\frac{|\gamma|}{2 \alpha}+k}\left|\partial_{x}^{2 \gamma} \partial_{t}^{k+1} R_{\alpha}\left(X_{m}, X_{j}\right)\right| \\
& \leq C \sum_{j}\left|\lambda_{j}\right| t_{j} \sum_{m \neq j} \frac{t_{m}^{\frac{n}{2 \alpha}+1}}{\left(t_{m}+t_{j}+\left|x_{m}-x_{j}\right|^{2 \alpha}\right)^{\frac{n}{2 \alpha}+2}} \\
& \leq C \sum_{j}\left|\lambda_{j}\right| t_{j} \sum_{m \neq j} \int_{S_{\delta / 2}^{(\alpha)}\left(X_{m}\right)} \frac{1}{\left(r+t_{j}+\left|z-x_{j}\right|^{2 \alpha}\right)^{\frac{n}{2 \alpha}+2}} d V(z, r) \\
& \leq C \sum_{j}\left|\lambda_{j}\right| t_{j} \int_{H \backslash S_{\delta}^{(\alpha)}\left(X_{j}\right)} \frac{1}{\left(r+t_{j}+\left|z-x_{j}\right|^{2 \alpha}\right)^{\frac{n}{2 \alpha}+2}} d V(z, r) \\
& =C \sum_{j}\left|\lambda_{j}\right| t_{j} \int_{H \backslash S_{\delta}^{(\alpha)}(0,1)} \frac{1}{\left(t_{j} t+t_{j}+\left|t_{j}^{\frac{1}{2 \alpha}} x+x_{j}-x_{j}\right|^{2 \alpha}\right)^{\frac{n}{2 \alpha}+2}} t_{j}^{\frac{n}{2 \alpha}+1} d V(x, t) \\
& =C \sum_{j}\left|\lambda_{j}\right| \int_{H \backslash S_{\delta}^{(\alpha)}(0,1)} \frac{1}{\left(t+1+|x|^{2 \alpha}\right)^{\frac{n}{2 \alpha}+2}} d V(x, t),
\end{aligned}
$$

where $C$ is independent of $\delta$. By Lemma 2, there exists a positive number $\delta_{0}$ such that if $\delta \geq \delta_{0}$ then $\left\|T_{1}^{\gamma, k} U_{1}^{\gamma, 1}+2 d_{\gamma, k+1} I\right\|<2\left|d_{\gamma, k+1}\right|$. Hence, we obtain $T_{1}^{\gamma, k} U_{1}^{\gamma, 1}$ is invertible, and thus $T_{1}^{\gamma, k}$ is onto whenever $\delta \geq \delta_{0}$.

\section{Interpolating Sequences of $\tilde{\mathcal{B}}_{\alpha}$ and $\tilde{\mathcal{B}}_{\alpha, 0}$}

In this section, as a limiting case of $\boldsymbol{b}_{\alpha}^{p}(p \rightarrow \infty)$, we give interpolation theorems for $\alpha$-parabolic Bloch spaces and $\alpha$-parabolic little Bloch spaces. We recall definitions of the $\alpha$-parabolic Bloch space and the $\alpha$-parabolic little Bloch space. (For details concerning these spaces, see Sections 7 and 9 of [7].) We denote by $\mathcal{B}_{\alpha}$ the set of all $L^{(\alpha)}$-harmonic and $C^{1}$-class functions $u$ on $H$ such that

$$
\|u\|_{\mathcal{B}_{\alpha}}=\left|u\left(Z_{0}\right)\right|+\sup _{(x, t) \in H}\left\{t^{\frac{1}{2 \alpha}}\left|\nabla_{x} u(x, t)\right|+t\left|\partial_{t} u(x, t)\right|\right\}<\infty,
$$

where $\nabla_{x}$ denotes the gradient operator and $Z_{0}=(0, \ldots, 0,1) \in H$. Let $\widetilde{\mathcal{B}}_{\alpha}$ denote the space of all function $u \in \mathcal{B}_{\alpha}$ such that $u\left(Z_{0}\right)=0$, and we call $\widetilde{\mathcal{B}}_{\alpha}$ the $\alpha$-parabolic Bloch space. It is known that $\widetilde{\mathcal{B}}_{\alpha} \subset C^{\infty}$ and $\widetilde{\mathcal{B}}_{\alpha}$ is a Banach space under the $\alpha$ parabolic Bloch norm $\|\cdot\|_{\mathcal{B}_{\alpha}}$ (see Theorems 7.3 and 7.4 of [7], respectively). We put

$$
\widetilde{\mathcal{B}}_{\alpha, 0}:=\left\{u \in \widetilde{\mathcal{B}}_{\alpha} ; \lim _{(x, t) \rightarrow \partial H \cup\{\infty\}}\left\{t^{\frac{1}{2 \alpha}}\left|\nabla_{x} u(x, t)\right|+t\left|\partial_{t} u(x, t)\right|\right\}=0\right\} .
$$

This is a closed subspace of $\widetilde{\mathcal{B}}_{\alpha}$ and hence a Banach space with $\alpha$-parabolic Bloch norm. We call $\widetilde{\mathcal{B}}_{\alpha, 0}$ the $\alpha$-parabolic little Bloch space.

Now let $\mathbb{X}=\left\{X_{j}\right\}=\left\{\left(x_{j}, t_{j}\right)\right\}$ be a sequence in $H,(\gamma, k) \in \mathbb{N}_{0}^{n} \times \mathbb{N}_{0} \backslash\{(0,0)\}$, and $u \in \widetilde{\mathcal{B}}_{\alpha}$. Define a sequence of real numbers $T_{\infty}^{\gamma, k} u$ by

$$
T_{\infty}^{\gamma, k} u=T_{\infty, \mathbb{X}}^{\gamma, k} u=\left\{t_{j}^{\frac{|\gamma|}{2 \alpha}+k} \partial_{x}^{\gamma} \partial_{t}^{k} u\left(X_{j}\right)\right\}
$$


We say that $\left\{X_{j}\right\}$ is a $\widetilde{\mathcal{B}}_{\alpha}$-interpolating sequence of order $(\gamma, k)$ if $T_{\infty}^{\gamma, k}: \widetilde{\mathcal{B}}_{\alpha} \rightarrow \ell^{\infty}$ is bounded and onto. We also say that $\left\{X_{j}\right\}$ is a $\widetilde{\mathcal{B}}_{\alpha, 0}$-interpolating sequence of $\operatorname{order}(\gamma, k)$ if $T_{\infty}^{\gamma, k}: \widetilde{\mathcal{B}}_{\alpha, 0} \rightarrow c_{0}$ is bounded and onto, where $c_{0}$ is the subspace of $\ell^{\infty}$ consisting of sequences that converge to 0 . We may regard a finite sequence as an element of $c_{0}$.

We first note that $T_{\infty}^{\gamma, k}: \widetilde{\mathcal{B}}_{\alpha} \rightarrow \ell^{\infty}$ is always bounded, which is given by the following lemma.

Lemma 7 ([7, Theorem 7.3]) Let $(\gamma, k) \in \mathbb{N}_{0}^{n} \times \mathbb{N}_{0} \backslash\{(0,0)\}$. Then, there exists a constant $C>0$ such that

$$
\left|\partial_{x}^{\gamma} \partial_{t}^{k} u(x, t)\right| \leq C t^{-\left(\frac{|\gamma|}{2 \alpha}+k\right)}\|u\|_{\mathcal{B}_{\alpha}}
$$

for all $u \in \widetilde{\mathcal{B}}_{\alpha}$ and $(x, t) \in H$.

Next we remark that $T_{\infty}^{\gamma, k}$ maps $\widetilde{\mathcal{B}}_{\alpha, 0}$ into $c_{0}$ whenever $\left\{X_{j}\right\}$ is $\delta$-separated in the $\alpha$-parabolic sense. In fact, it derives from the following lemma, because $\left\{X_{j}\right\}$ diverges from any compact set of $H$.

Lemma 8 Let $(\gamma, k) \in \mathbb{N}_{0}^{n} \times \mathbb{N}_{0} \backslash\{(0,0)\}$ and $u \in \widetilde{\mathcal{B}}_{\alpha, 0}$. Then,

$$
\lim _{(x, t) \rightarrow \partial H \cup\{\infty\}} t^{\frac{|\gamma|}{2 \alpha}+k} \partial_{x}^{\gamma} \partial_{t}^{k} u(x, t)=0 .
$$

To prove this lemma, we use the modified $\alpha$-parabolic Bergman kernel $\widetilde{R}_{\alpha}(X, Y)$, which is defined in Section 7 of [7]:

$$
\widetilde{R}_{\alpha}(X, Y):=R_{\alpha}(X, Y)-R_{\alpha}\left(Z_{0}, Y\right)
$$

where $Z_{0}=(1,0, \cdots, 0)$. Then $\widetilde{R}_{\alpha}(X, \cdot) \in \boldsymbol{b}_{\alpha}^{1}$ by Lemma 7.5 of [7]. It was shown in Theorem 7.9 of [7] that

$$
u(x, t)=c_{\ell} \int_{H} s^{\ell} \partial_{s}^{\ell} u(y, s) \widetilde{R}_{\alpha}(x, t ; y, s) d V(y, s)
$$

for all $u \in \widetilde{\mathcal{B}}_{\alpha}$ and $\ell \in \mathbb{N}$, where $c_{\ell}=(-2)^{\ell} / \ell !$.

Now we return to the proof of Lemma 8 . The argument here is inspired by [14].

Proof of Lemma 8 Let $u \in \widetilde{\mathcal{B}}_{\alpha, 0}$. Then, by Eq. 21 with $\ell=1$, we have

$$
\partial_{x}^{\gamma} \partial_{t}^{k} u(x, t)=4 \int_{H} s \partial_{s} u(y, s) \partial_{x}^{\gamma} \partial_{t}^{k+1} W^{(\alpha)}(x-y, t+s) d V(y, s)
$$


for all $(x, t) \in H$. Given $\varepsilon>0$, there exists a compact set $K \subset H$ such that $\left|s \partial_{s} u(y, s)\right|<\varepsilon$ for all $(y, s) \in K^{c}$, because $u \in \widetilde{\mathcal{B}}_{\alpha, 0}$. Hence if $k \neq 0$ or $\gamma \neq 0$, then Eq. 4 and Lemma 2 imply that for all $(x, t) \in H$,

$$
\begin{aligned}
t^{\frac{|\gamma|}{2 \alpha}+k}\left|\partial_{x}^{\gamma} \partial_{t}^{k} u(x, t)\right| \leq & 4 t^{\frac{|\gamma|}{2 \alpha}+k} \int_{K^{c}} s\left|\partial_{s} u(y, s)\right| \cdot\left|\partial_{x}^{\gamma} \partial_{t}^{k+1} W^{(\alpha)}(x-y, t+s)\right| d V(y, s) \\
& +4 t^{\frac{|\gamma|}{2 \alpha}+k} \int_{K} s\left|\partial_{s} u(y, s)\right| \cdot\left|\partial_{x}^{\gamma} \partial_{t}^{k+1} W^{(\alpha)}(x-y, t+s)\right| d V(y, s) \\
\leq & C \varepsilon t \int_{K^{c}} \frac{1}{\left(t+s+|x-y|^{2 \alpha}\right)^{\frac{n}{2 \alpha}+2}} d V(y, s) \\
& +C\|u\|_{\mathcal{B}_{\alpha}} \cdot t \int_{K} \frac{1}{\left(t+s+|x-y|^{2 \alpha}\right)^{\frac{n}{2 \alpha}+2}} d V(y, s) \\
\leq & C \varepsilon+C\|u\|_{\mathcal{B}_{\alpha}} \frac{t}{\left(1+t+|x|^{2 \alpha}\right)^{\frac{n}{2 \alpha}+2}} .
\end{aligned}
$$

Therefore, we have

$$
\lim _{(x, t) \rightarrow \partial H \cup\{\infty\}} t^{\frac{|\gamma|}{2 \alpha}+k}\left|\partial_{x}^{\gamma} \partial_{t}^{k} u(x, t)\right| \leq C \varepsilon
$$

For a multi-index $\gamma \in \mathbb{N}_{0}^{n}$ and $k \in \mathbb{N}_{0}$, we also use the kernel $\widetilde{R}_{\alpha}^{\gamma, k}(X, Y)$ defined by

$$
\widetilde{R}_{\alpha}^{\gamma, k}(X, Y):=R_{\alpha}^{\gamma, k}(X, Y)-R_{\alpha}^{\gamma, k}\left(Z_{0}, Y\right)
$$

where $Z_{0}=(0,1)$. We need the following estimate of $\widetilde{R}_{\alpha}^{\gamma, k}(X, Y)=\widetilde{R}_{\alpha}^{\gamma, k}(x, t ; y, s)$.

Lemma 9 Let $K$ be a compact subset of $H, \gamma \in \mathbb{N}_{0}^{n}, k \in \mathbb{N}_{0}$, and $0<\delta<1$. Then, there exists a constant $C=C(n, \alpha, \gamma, k, \delta, K)>0$ such that

$$
\begin{aligned}
& s^{\frac{n}{2 \alpha}+1}\left|\widetilde{R}_{\alpha}^{\gamma, k}(x, t ; y, s)\right| \\
& \quad \leq C\left(\int_{S_{\delta}^{(\alpha)}(y, s)} \frac{1}{\left(1+r+|z|^{2 \alpha}\right)^{\frac{n}{2 \alpha}+2}} d V(z, r)+\int_{S_{\delta}^{(\alpha)}(y, s)} \frac{1}{\left(1+r+|z|^{2 \alpha}\right)^{\frac{n+1}{2 \alpha}+1}} d V(z, r)\right)
\end{aligned}
$$

for all $(x, t) \in K$ and $(y, s) \in H$.

Proof The equality

$$
\begin{aligned}
R_{\alpha}^{\gamma, k} & (x, t ; y, s)-R_{\alpha}^{\gamma, k}(x, 1 ; y, s) \\
& =-2 c_{k} s^{\frac{|\gamma|}{2 \alpha}+k}\left(\partial_{x}^{\gamma} \partial_{t}^{k+1} W^{(\alpha)}(x-y, t+s)-\partial_{x}^{\gamma} \partial_{t}^{k+1} W^{(\alpha)}(x-y, 1+s)\right) \\
& =-2 c_{k} s^{\frac{|\gamma|}{2 \alpha}+k} \int_{1}^{t} \partial_{x}^{\gamma} \partial_{t}^{k+2} W^{(\alpha)}(x-y, \tau+s) d \tau
\end{aligned}
$$


and Eq. 4 imply that

$$
\begin{aligned}
\left|R_{\alpha}^{\gamma, k}(x, t ; y, s)-R_{\alpha}^{\gamma, k}(x, 1 ; y, s)\right| & \leq C_{1} s^{\frac{|\gamma|}{2 \alpha}+k}\left|\int_{1}^{t} \frac{1}{\left(\tau+s+|x-y|^{2 \alpha}\right)^{\frac{n+|\gamma|}{2 \alpha}}+k+2} d \tau\right| \\
& \leq \frac{C_{1} s^{\frac{|\gamma|}{2 \alpha}+k}}{\left(1+s+|x-y|^{2 \alpha}\right)^{\frac{n+|\gamma|}{2 \alpha}+k+2}} \\
& \leq \frac{C_{1}}{\left(1+s+|y|^{2 \alpha}\right)^{\frac{n}{2 \alpha}+2}}
\end{aligned}
$$

for all $(x, t) \in K$ and $(y, s) \in H$. The equality

$$
\begin{aligned}
& R_{\alpha}^{\gamma, k}(x, 1 ; y, s)-R_{\alpha}^{\gamma, k}(0,1 ; y, s) \\
& \quad=-2 c_{k} s^{\frac{|\gamma|}{2 \alpha}+k}\left(\partial_{x}^{\gamma} \partial_{t}^{k+1} W^{(\alpha)}(x-y, 1+s)-\partial_{x}^{\gamma} \partial_{t}^{k+1} W^{(\alpha)}(-y, 1+s)\right) \\
& \quad=-2 c_{k} s^{\frac{|\gamma|}{2 \alpha}+k} \int_{0}^{1} x \cdot \nabla_{x} \partial_{x}^{\gamma} \partial_{t}^{k+1} W^{(\alpha)}(\sigma x-y, 1+s) d \sigma
\end{aligned}
$$

and Eq. 4 imply that

$$
\begin{aligned}
\left|R_{\alpha}^{\gamma, k}(x, 1 ; y, s)-R_{\alpha}^{\gamma, k}(0,1 ; y, s)\right| & \leq C_{2} s^{\frac{|\gamma|}{2 \alpha}+k}|x| \int_{0}^{1}\left|\nabla_{x} \partial_{x}^{\gamma} \partial_{t}^{k+1} W^{(\alpha)}(\sigma x-y, 1+s)\right| d \sigma \\
& \leq C_{2} s^{\frac{|\gamma|}{2 \alpha}+k}|x| \int_{0}^{1} \frac{1}{\left(1+s+|\sigma x-y|^{2 \alpha}\right)^{\frac{n+|\gamma|+1}{2 \alpha}+k+1}} d \sigma \\
& \leq C_{2} \frac{1}{\left(1+s+|y|^{2 \alpha}\right)^{\frac{n+1}{2 \alpha}+1}}
\end{aligned}
$$

for all $(x, t) \in K$ and $(y, s) \in H$. Therefore, we have

$$
\begin{aligned}
& \left|\widetilde{R}_{\alpha}^{\gamma, k}(x, t ; y, s)\right| \\
& \quad \leq\left|R_{\alpha}^{\gamma, k}(x, t ; y, s)-R_{\alpha}^{\gamma, k}(x, 1 ; y, s)\right|+\left|R_{\alpha}^{\gamma, k}(x, 1 ; y, s)-R_{\alpha}^{\gamma, k}(0,1 ; y, s)\right| \\
& \quad \leq \frac{C_{1}}{\left(1+s+|y|^{2 \alpha}\right)^{\frac{n}{2 \alpha}+2}}+\frac{C_{2}}{\left(1+s+|y|^{2 \alpha}\right)^{\frac{n+1}{2 \alpha}+1}}
\end{aligned}
$$

for all $(x, t) \in K$ and $(y, s) \in H$. As in the proof of Lemma 1 , for each $k_{1}, k_{2} \in \mathbb{N}_{0}$ there exists a constant $C>0$ such that

$$
\frac{s^{\frac{n}{2 \alpha}+1}}{\left(1+s+|y|^{2 \alpha}\right)^{\frac{n+k_{1}}{2 \alpha}+k_{2}+1}} \leq C \int_{S_{\delta}^{(\alpha)}(y, s)} \frac{1}{\left(1+r+|z|^{2 \alpha}\right)^{\frac{n+k_{1}}{2 \alpha}+k_{2}+1}} d V(z, r)
$$

for all $Y=(y, s) \in H$. Thus, the lemma follows from Eqs. 22 and 23.

Let $\gamma \in \mathbb{N}_{0}^{n}$ and $k \in \mathbb{N}_{0}$. As in Section 4, the following operator $U_{\infty}^{\gamma, k}$ plays an important role in the theory of interpolation sequences for $\alpha$-parabolic Bloch spaces and $\alpha$-parabolic little Bloch spaces. For $\left\{\lambda_{j}\right\} \in \ell^{\infty}$, let

$$
\left(U_{\infty}^{\gamma, k}\left\{\lambda_{j}\right\}\right)(X)=\left(U_{\infty, \mathbb{X}}^{\gamma, k}\left\{\lambda_{j}\right\}\right)(X):=\sum_{j} \lambda_{j} t_{j}^{\frac{n}{2 \alpha}+1} \widetilde{R}_{\alpha}^{\gamma, k}\left(X, X_{j}\right) \quad(X \in H) .
$$


We have the following lemma.

Lemma 10 Let $\gamma \in \mathbb{N}_{0}^{n}$ and $k \in \mathbb{N}_{0}$. Suppose that $\mathbb{X}=\left\{X_{j}\right\}=\left\{\left(x_{j}, t_{j}\right)\right\}$ is $\delta$-separated in the $\alpha$-parabolic sense. Then, the operator $U_{\infty}^{\gamma, k}=U_{\infty, \mathbb{X}}^{\gamma, k}: \ell^{\infty} \rightarrow \widetilde{\mathcal{B}}_{\alpha}$ is bounded and $U_{\infty}^{\gamma, k}$ maps $c_{0}$ into $\widetilde{\mathcal{B}}_{\alpha, 0}$.

Proof Let $\left\{\lambda_{j}\right\} \in \ell^{\infty}$. We begin with showing that the series Eq. 24 converges uniformly on compact subsets of $H$ (we only use the pointwise convergence of this series later). Let $K$ be a compact subset of $H$. Lemma 9 implies that

$$
\begin{aligned}
\left|\lambda_{j} t_{j}^{\frac{n}{2 \alpha}+1} \widetilde{R}_{\alpha}^{\gamma, k}\left(X, X_{j}\right)\right| \leq C\left\|\left\{\lambda_{j}\right\}\right\|_{\infty} & \left(\int_{S_{\delta}^{(\alpha)}\left(X_{j}\right)} \frac{1}{\left(1+r+|z|^{2 \alpha}\right)^{\frac{n}{2 \alpha}+2}} d V(z, r)\right. \\
& \left.+\int_{S_{\delta}^{(\alpha)}\left(X_{j}\right)} \frac{1}{\left(1+r+|z|^{2 \alpha}\right)^{\frac{n+1}{2 \alpha}+1}} d V(z, r)\right)
\end{aligned}
$$

for all $X=(x, t) \in K$, and Lemma 2 shows

$$
\sum_{j} \int_{S_{\delta}^{(\alpha)}\left(X_{j}\right)} \frac{1}{\left(1+r+|z|^{2 \alpha}\right)^{\frac{n}{2 \alpha}+2}} d V(z, r) \leq \int_{H} \frac{1}{\left(1+r+|z|^{2 \alpha}\right)^{\frac{n}{2 \alpha}+2}} d V(z, r)<\infty
$$

and

$$
\sum_{j} \int_{S_{\delta}^{(\alpha)}\left(X_{j}\right)} \frac{1}{\left(1+r+|z|^{2 \alpha}\right)^{\frac{n+1}{2 \alpha}+1}} d V(z, r) \leq \int_{H} \frac{1}{\left(1+r+|z|^{2 \alpha}\right)^{\frac{n+1}{2 \alpha}+1}} d V(z, r)<\infty,
$$

hence the series Eq. 24 converges uniformly on $K$.

Put

$$
u_{N}(X)=\sum_{j=1}^{N} \lambda_{j} t_{j}^{\frac{n}{2 \alpha}+1} \widetilde{R}_{\alpha}^{\gamma, k}\left(X, X_{j}\right), \quad X \in H .
$$

We claim that $\left\{u_{N}\right\}$ is bounded in $\widetilde{\mathcal{B}}_{\alpha}$. In fact, as in the proof of Lemma 1, for each $\left(\gamma^{\prime}, \ell\right) \in \mathbb{N}_{0}^{n} \times \mathbb{N}_{0} \backslash\{(0,0)\}$ we have

$$
\begin{aligned}
& \sum_{j=1}^{N}\left|\lambda_{j}\right| t_{j}^{\frac{n}{2 \alpha}+1}\left|\partial_{x}^{\gamma^{\prime}} \partial_{t}^{\ell} \widetilde{R}_{\alpha}^{\gamma, k}\left(X, X_{j}\right)\right|=\sum_{j=1}^{N}\left|\lambda_{j}\right| t_{j}^{\frac{n}{2 \alpha}+1}\left|\partial_{x}^{\gamma^{\prime}} \partial_{t}^{\ell} R_{\alpha}^{\gamma, k}\left(X, X_{j}\right)\right| \\
& \quad \leq C\left(\sup _{1 \leq j \leq N}\left|\lambda_{j}\right|\right) \sum_{j=1}^{N} \int_{S_{\delta}^{(\alpha)}\left(X_{j}\right)} \frac{1}{\left(t+r+|x-z|^{2 \alpha}\right)^{\frac{n+\left|\gamma^{\prime}\right|}{2 \alpha}+\ell+1}} d V(z, r)
\end{aligned}
$$

for all $X=(x, t) \in H$. Therefore, Eq. 25 and Lemma 2 also imply that

$$
\sum_{j=1}^{N}\left|\lambda_{j}\right| t_{j}^{\frac{n}{2 \alpha}+1}\left|\partial_{t} \widetilde{R}_{\alpha}^{\gamma, k}\left(X, X_{j}\right)\right| \leq C t^{-1}\left(\sup _{1 \leq j \leq N}\left|\lambda_{j}\right|\right)
$$

and

$$
\sum_{j=1}^{N}\left|\lambda_{j}\right| t_{j}^{\frac{n}{2 \alpha}+1}\left|\partial_{x_{\ell}} \widetilde{R}_{\alpha}^{\gamma, k}\left(X, X_{j}\right)\right| \leq C t^{-\frac{1}{2 \alpha}}\left(\sup _{1 \leq j \leq N}\left|\lambda_{j}\right|\right)
$$


for all $X=(x, t) \in H$, which shows $\left\|u_{N}\right\|_{\mathcal{B}_{\alpha}} \leq C\left\|\left\{\lambda_{j}\right\}\right\|_{\infty}$ for all $N \in \mathbb{N}$.

Here we recall the fact that the dual space of $\boldsymbol{b}_{\alpha}^{1}$ can be identified with $\widetilde{\mathcal{B}}_{\alpha}$ under the pairing

$$
\langle u, v\rangle=-2 \int_{H} s \partial_{s} u(y, s) v(y, s) d V(y, s), \quad u \in \widetilde{\mathcal{B}}_{\alpha}, v \in \boldsymbol{b}_{\alpha}^{1}
$$

(see Theorem 8.4 of [7]). Moreover, since $L^{1}$ is separable and there exists a bounded projection $P$ from $L^{1}$ onto $\boldsymbol{b}_{\alpha}^{1}$ by (2) of Theorem 6.4 of [7], $\boldsymbol{b}_{\alpha}^{1}$ is also separable. Therefore, the Banach-Alaoglu theorem implies that there exists a subsequence $\left\{u_{N_{i}}\right\} \subset\left\{u_{N}\right\}$ and $u \in \widetilde{\mathcal{B}}_{\alpha}$ such that $\left\{u_{N_{i}}\right\}$ converges to $u$ in the $\mathrm{w}^{*}$-topology. Hence, since $\widetilde{R}_{\alpha}(X, \cdot) \in \boldsymbol{b}_{\alpha}^{1}$ for each $X \in H$, Eq. 21 with $\ell=1$ shows

$$
u(X)=\left\langle u, \widetilde{R}_{\alpha}(X, \cdot)\right\rangle=\lim _{i}\left\langle u_{N_{i}}, \widetilde{R}_{\alpha}(X, \cdot)\right\rangle=\lim _{i} u_{N_{i}}(X)=\left(U_{\infty}^{\gamma, k}\left\{\lambda_{j}\right\}\right)(X) .
$$

This implies $U_{\infty}^{\gamma, k}\left\{\lambda_{j}\right\} \in \widetilde{\mathcal{B}}_{\alpha}$ and $\left\|U_{\infty}^{\gamma, k}\left\{\lambda_{j}\right\}\right\|_{\mathcal{B}_{\alpha}} \leq \liminf \left\|u_{N_{i}}\right\|_{\mathcal{B}_{\alpha}} \leq C\left\|\left\{\lambda_{j}\right\}\right\|_{\infty}$, that is, the operator $U_{\infty}^{\gamma, k}: \ell^{\infty} \rightarrow \widetilde{\mathcal{B}}_{\alpha}$ is bounded.

Next let $\left\{\lambda_{j}^{\prime}\right\} \in c_{0}$ and put

$$
v_{N}(X)=\sum_{j=1}^{N} \lambda_{j}^{\prime} t_{j}^{\frac{n}{2 \alpha}+1} \widetilde{R}_{\alpha}^{\gamma, k}\left(X, X_{j}\right), \quad X \in H .
$$

Then, Eq. 4 implies that $v_{N} \in \widetilde{\mathcal{B}}_{\alpha, 0}$. Moreover, Eq. 26 and Eq. 27 show that

$$
\left\|v_{M}-v_{N}\right\|_{\mathcal{B}_{\alpha}} \leq C\left(\sup _{N+1 \leq j \leq M}\left|\lambda_{j}^{\prime}\right|\right) \rightarrow 0 \quad(M \geq N \rightarrow \infty) .
$$

Hence, there exists a function $v \in \widetilde{\mathcal{B}}_{\alpha, 0}$ such that $\left\{v_{N}\right\}$ converges to $v$ in $\widetilde{\mathcal{B}}_{\alpha}$. Therefore, Proposition 7.2 of [7] implies that

$$
v(X)=\lim _{N} v_{N}(X)=\left(U_{\infty}^{\gamma, k}\left\{\lambda_{j}^{\prime}\right\}\right)(X),
$$

which shows $U_{\infty}^{\gamma, k}$ maps $c_{0}$ into $\widetilde{\mathcal{B}}_{\alpha, 0}$.

Remark 2 Let $\left(\gamma^{\prime}, \ell\right) \in \mathbb{N}_{0}^{n} \times \mathbb{N}_{0} \backslash\{(0,0)\}$. Then $\left\{\partial_{x}^{\gamma^{\prime}} \partial_{t}^{\ell} u_{N}\right\}$ converges uniformly on $\mathbb{R}^{n} \times[\tau, \infty)$ for every $\tau>0$. In fact, Eq. 25 and Lemma 2 give us

$$
\begin{aligned}
& \sum_{j=1}^{N}\left|\lambda_{j}\right| t_{j}^{\frac{n}{2 \alpha}+1}\left|\partial_{x}^{\gamma^{\prime}} \partial_{t}^{\ell} \widetilde{R}_{\alpha}^{\gamma, k}\left(X, X_{j}\right)\right| \\
& \quad \leq C\left\|\left\{\lambda_{j}\right\}\right\|_{\infty} \sum_{j=1}^{N} \int_{S_{\delta}^{(\alpha)}\left(X_{j}\right)} \frac{1}{\left(t+r+|x-z|^{2 \alpha}\right)^{\frac{n+\left|\gamma^{\prime}\right|}{2 \alpha}+\ell+1}} d V(z, r) \\
& \quad \leq C t^{-\frac{\left|\gamma^{\prime}\right|}{2 \alpha}-\ell}
\end{aligned}
$$

for all $X \in H$. This shows our desired assertion immediately.

We state the interpolation theorems for $\alpha$-parabolic Bloch spaces and $\alpha$-parabolic little Bloch spaces. 
Theorem 3 Let $(\gamma, k) \in \mathbb{N}_{0}^{n} \times \mathbb{N}_{0} \backslash\{(0,0)\}$. Then, there exists a positive number $\delta_{0}$ such that if $\left\{X_{j}\right\}$ is $\delta$-separated in the $\alpha$-parabolic sense with $\delta \geq \delta_{0}$ then $\left\{X_{j}\right\}$ is a $\widetilde{\mathcal{B}}_{\alpha^{-}}$ interpolating and $\widetilde{\mathcal{B}}_{\alpha, 0}$-interpolating sequence of order $(\gamma, k)$.

Proof We suppose that $\left\{X_{j}\right\}=\left\{\left(x_{j}, t_{j}\right)\right\}$ is $\delta$-separated in the $\alpha$-parabolic sense. By Lemma 10 , the operator $U_{\infty}^{\gamma, 0}: \ell^{\infty} \rightarrow \widetilde{\mathcal{B}}_{\alpha}$ is bounded and $U_{\infty}^{\gamma, 0}$ maps $c_{0}$ into $\widetilde{\mathcal{B}}_{\alpha, 0}$. Therefore, the operator $T_{\infty}^{\gamma, k} U_{\infty}^{\gamma, 0}: \ell^{\infty} \rightarrow \ell^{\infty}$ is also bounded and maps $c_{0}$ into $c_{0}$ by Lemmas 7 and 8 . As in the proof of Theorem 2, it suffices to show that there exists a positive number $\delta_{0}$ such that if $\delta \geq \delta_{0}$ then $\left\|T_{\infty}^{\gamma, k} U_{\infty}^{\gamma, 0}-d_{\gamma, k} I\right\|<\left|d_{\gamma, k}\right|$, where $I$ is the identity operator on $\ell^{\infty}$ and $d_{\gamma, k}=s^{\frac{n+2|\gamma|}{2 \alpha}+k+1} \partial_{x}^{2 \gamma} \partial_{t}^{k} R_{\alpha}(y, s ; y, s)$.

The operator $T_{\infty}^{\gamma, k} U_{\infty}^{\gamma, 0}-d_{\gamma, k} I$ maps a sequence $\left\{\lambda_{j}\right\}$ in $\ell^{\infty}$ to a sequence $\left\{\xi_{m}\right\}$ in $\ell^{\infty}$ given by

$$
\xi_{m}=t_{m}^{\frac{|\gamma|}{2 \alpha}+k}\left(\partial_{x}^{\gamma} \partial_{t}^{k} U_{\infty}^{\gamma, 0}\left\{\lambda_{j}\right\}\right)\left(X_{m}\right)-d_{\gamma, k} \lambda_{m}
$$

By Remark 2, we can differentiate term by term, so that

$$
\begin{aligned}
\xi_{m} & =t_{m}^{\frac{|\gamma|}{2 \alpha}+k} \sum_{j} \lambda_{j} t_{j}^{\frac{n}{2 \alpha}+1} \partial_{x}^{\gamma} \partial_{t}^{k} \widetilde{R}_{\alpha}^{\gamma, 0}\left(X_{m}, X_{j}\right)-d_{\gamma, k} \lambda_{m} \\
& =t_{m}^{\frac{|\gamma|}{2 \alpha}+k} \sum_{j \neq m} \lambda_{j} t_{j}^{\frac{n+|\gamma|}{2 \alpha}+1} \partial_{x}^{2 \gamma} \partial_{t}^{k} R_{\alpha}\left(X_{m}, X_{j}\right)
\end{aligned}
$$

Since $1 / 3 \leq t_{j} / r \leq 3$ for $(z, r) \in S_{\delta / 2}^{(\alpha)}\left(X_{j}\right)$, Eq. 4 and the proof of Lemma 1 show that

$$
\begin{aligned}
\left|\xi_{m}\right| & \leq t_{m}^{\frac{|\gamma|}{2 \alpha}+k} \sum_{j \neq m}\left|\lambda_{j}\right| t_{j}^{\frac{n+|\gamma|}{2 \alpha}+1}\left|\partial_{x}^{2 \gamma} \partial_{t}^{k} R_{\alpha}\left(X_{m}, X_{j}\right)\right| \\
& \leq C t_{m}^{\frac{|\gamma|}{2 \alpha}+k} \sum_{j \neq m} \frac{\left|\lambda_{j}\right| t_{j}^{\frac{n+|\gamma|}{2 \alpha}+1}}{\left(t_{m}+t_{j}+\left|x_{m}-x_{j}\right|^{2 \alpha}\right)^{\frac{n+2|\gamma|}{2 \alpha}+k+1}} \\
& \leq C\left\|\left\{\lambda_{j}\right\}\right\|_{\infty} t_{m}^{\frac{|\gamma|}{2 \alpha}+k} \sum_{j \neq m} \int_{S_{\delta / 2}^{(\alpha)}\left(X_{j}\right)} \frac{1}{\left(t_{m}+r+\left|x_{m}-z\right|^{2 \alpha}\right)^{\frac{n+|\gamma|}{2 \alpha}+k+1}} d V(z, r) \\
& \leq C\left\|\left\{\lambda_{j}\right\}\right\|_{\infty} t_{m}^{\frac{|\gamma|}{2 \alpha}+k} \int_{H \backslash S_{\delta}^{(\alpha)}\left(X_{m}\right)} \frac{1}{\left(t_{m}+r+\left|x_{m}-z\right|^{2 \alpha}\right)^{\frac{n+|\gamma|}{2 \alpha}+k+1}} d V(z, r) \\
& =C\left\|\left\{\lambda_{j}\right\}\right\|_{\infty} t_{m}^{\frac{|\gamma|}{2 \alpha}+k} \int_{H \backslash S_{\delta}^{(\alpha)}(0,1)} \frac{1}{\left(t_{m}+t_{m} t+\left|x_{m}-\left(t_{m}^{\frac{1}{2 \alpha}} x+x_{m}\right)\right|^{2 \alpha}\right)^{\frac{n+|\gamma|}{2 \alpha}+k+1}} t_{m}^{\frac{n}{2 \alpha}+1} d V(x, t) \\
& =C\left\|\left\{\lambda_{j}\right\}\right\|_{\infty} \int_{H \backslash S_{\delta}^{(\alpha)}(0,1)} \frac{1}{\left(1+t+|x|^{2 \alpha}\right)^{\frac{n+|\gamma|}{2 \alpha}+k+1}} d V(x, t) .
\end{aligned}
$$

Hence, as in the proof of Theorem 2, Lemma 2 implies that there exists a positive number $\delta_{0}$ such that if $\delta \geq \delta_{0}$ then $\left\|T_{\infty}^{\gamma, k} U_{\infty}^{\gamma, 0}-d_{\gamma, k} I\right\|<\left|d_{\gamma, k}\right|$. 
Open Access This article is distributed under the terms of the Creative Commons Attribution Noncommercial License which permits any noncommercial use, distribution, and reproduction in any medium, provided the original author(s) and source are credited.

\section{References}

1. Amar, E.: Suites d'interpolation pour les classes de Bergman de la boule du polydisque de $\mathbb{C}^{n}$. Canad. J. Math. 30, 711-737 (1978)

2. Axler, S., Bourdon, P., Ramey, W.: Harmonic Function Theory. Springer, New York (1992)

3. Choe, B.R. Yi, H.: Representations and interpolations of harmonic Bergman functions on halfspaces. Nagoya Math. J. 151, 51-89 (1998)

4. Choe, B.R., Koo, H. Yi, H.: Positive Toeplitz operators between the harmonic Bergman spaces. Potential Anal. 17, 307-335 (2002)

5. Luecking, D.: Multipliers of Bergman spaces into Lebesgue spaces. Proc. Edinburgh Math. Soc. 29, 125-131 (1986)

6. Marco, N., Massaneda, X.: On density conditions for interpolation in the ball. Canad. Math. Bull. 46, 559-574 (2003)

7. Nishio, M., Shimomura, K., Suzuki, N.: $\alpha$-parabolic Bergman spaces. Osaka J. Math. 42, 133-162 (2005)

8. Nishio, M., Shimomura, K., Suzuki, N.: $L^{p}$-boundedness of Bergman projections for $\alpha$-parabolic operators. Adv. Stud. Pure Math. 44, 305-318 (2006)

9. Nishio, M., Suzuki, N., Yamada, M.: Toeplitz operators and Carleson measures on parabolic Bergman spaces. Hokkaido Math. J. 36, 563-583 (2007)

10. Nishio, M., Yamada, M.: Carleson type measures on parabolic Bergman spaces. J. Math. Soc. Japan 58, 83-96 (2006)

11. Ramey, W., Yi, H.: Harmonic Bergman functions on half-spaces. Trans. Amer. Math. Soc. 348, 633-660 (1996)

12. Rochberg, R.: Interpolation by functions in Bergman spaces. Michigan Math. J. 29, 229-236 (1982)

13. Seip, K.: Beurling type density theorems in the unit disk. Invent. Math. 113, 21-39 (1993)

14. Yi, H.: Harmonic little Bloch functions on half-spaces. Math. Japon. 47(1), 21-28 (1998)

15. Zhu, K.: Evaluation operators on the Bergman space. Math. Proc. Cambridge Philos. Soc. 117, 513-523 (1995) 